\title{
CD44 expression in endothelial colony-forming cells regulates neurovascular trophic effect
}

\author{
Susumu Sakimoto, ${ }^{1}$ Valentina Marchetti, ${ }^{2}$ Edith Aguilar, ${ }^{1}$ Kelsey Lee, ${ }^{2}$ Yoshihiko Usui, ${ }^{1}$ \\ Salome Murinello, ${ }^{1}$ Felicitas Bucher, ${ }^{1}$ Jennifer K. Trombley, ${ }^{3}$ Regis Fallon, ${ }^{1}$ Ravenska Wagey, ${ }^{2}$ \\ Carrie Peters, ${ }^{2}$ Elizabeth L. Scheppke, ${ }^{3}$ Peter D. Westenskow, ${ }^{3}$ and Martin Friedlander ${ }^{1}$ \\ 'Department of Cell and Molecular Biology, The Scripps Research Institute, La Jolla, California, USA. ${ }^{2}$ STEMCELL \\ Technologies, Vancouver, British Columbia, Canada. ${ }^{3}$ Lowy Medical Research Institute, La Jolla, California, USA.
}

\begin{abstract}
Vascular abnormalities are a common component of eye diseases that often lead to vision loss. Vaso-obliteration is associated with inherited retinal degenerations, since photoreceptor atrophy lowers local metabolic demands and vascular support to those regions is no longer required. Given the degree of neurovascular crosstalk in the retina, it may be possible to use one cell type to rescue another cell type in the face of severe stress, such as hypoxia or genetically encoded cell-specific degenerations. Here, we show that intravitreally injected human endothelial colony-forming cells (ECFCs) that can be isolated and differentiated from cord blood in xeno-free media collect in the vitreous cavity and rescue vaso-obliteration and neurodegeneration in animal models of retinal disease. Furthermore, we determined that a subset of the ECFCs was more effective at anatomically and functionally preventing retinopathy; these cells expressed high levels of CD44, the hyaluronic acid receptor, and IGFBPs (insulin-like growth factor-binding proteins). Injection of cultured media from ECFCs or only recombinant human IGFBPs also rescued the ischemia phenotype. These results help us to understand the mechanism of ECFC-based therapies for ischemic insults and retinal neurodegenerative diseases.
\end{abstract}

\section{Introduction}

Visual loss in retinal diseases is caused by damage to, and subsequent loss of, photoreceptors that are located in the outer retina. A variety of conditions can lead to retinal ischemia and subsequent pathological angiogenesis. The devastating consequences of retinal neovascularization are seen in diabetic retinopathy and age-related macular degeneration, major causes of vision loss in industrialized countries. Changes intiated by diseases characterized by pathological angiogenesis may extend to the outer layer of the retina where they can lead to secondary photoreceptor cell damage. In contrast, a group of inherited retinal degenerative diseases directly affect the photoreceptor cells (e.g., retinitis pigmentosa [RP]). Histologically, RP is characterized by widespread loss of photoreceptor cells, thinning of the outer retina, and atrophy of retinal vasculature (1). There have been no effective treatments to slow or reverse the progression of the photoreceptor loss. A randomized clinical trial of CNTF-transfected encapsulated ARPE-19 cells (NT-501) injected into the vitreous showed a dose-dependent increase in retinal thickness but no functional rescue for patients with RP (2).

Endothelial colony-forming cells (ECFCs) (3), a subset of endothelial progenitor cells (EPCs), are a potential source of autologous grafts for therapeutic clinical use. ECFCs can be isolated from human cord or peripheral blood and have robust clonal proliferative potential. They have been reported to home to the site of tissue ischemia after intravenous injection, where they improve circulation in a model of myocardial infarction (4), stroke (5), ischemic retinopathy $(6,7)$, and ischemic limb injury $(8,9)$. Although a paracrine trophic rescue effect of ECFCs has been postulated $(10,11)$, factors that may mediate this effect remain poorly characterized.

Hyaluronic acid (HA), which was initially named from "hyaloid (vitreous) and uronic acid," was isolated from the vitreous of bovine eyes in 1934 (12). The primary receptor for HA, CD44, is a ubiquitously expressed transmembrane glycoprotein. It is also a receptor for various extracellular matrix proteins, such as collagen and osteopontin (13). Beyond its role as an adhesion molecule, CD44 modulates cellular signaling (13-15) by forming coreceptor complexes with various receptor tyrosine kinases. Moreover, cells with a higher density of 
CD44 possess stem-like properties in normal and neoplastic tissue and home to specific tissue niches $(16,17)$. Based on a previous report showing a retinal rescue effect by CD44 myeloid progenitors (18), together with the fact that CD44 is a major receptor for HA, which is abundantly distributed in vitreous body, we sought to determine the regenerative capacity of CD44hi ECFCs in the oxygen-induced retinopathy (OIR) model.

In this study, we demonstrate that intravitreally injected ECFCs can reside in the vitreous and accelerate retinal vascular repair both morphologically and functionally in a murine model of ischemic retinopathy. We define a subpopulation of intravitreally injected ECFCs with the canonical HA receptor, CD44, that modulate retinal revascularization in both ischemic retinopathy and late-onset retinal degeneration. This establishes the paracrine effect of ECFCs and explains the mechanism of vascular repair. Gene expression analysis of injected ECFCs revealed that genes encoding several angiocrine growth factors were functionally upregulated and exogenous administration of insulin-like growth factor-binding proteins (IGFBPs) rescued OIR. Together, our results suggest that ECFC-based cell therapy is a therapeutic that has potential application to many types of retinal diseases.

\section{Results}

In vitro and in vivo characterization of ECFCs cultured with xeno-free media. Conventional methods for isolating and differentiating ECFCs utilize animal- and bacterial-derived reagents $(3,19)$. To improve the safety profile of ECFCs, we developed xeno-free media (XFM) and a cell attachment substrate for derivation and propagation for human ECFCs from cord blood. We observed no obvious differences in morphology or colony derivation efficiency of ECFCs isolated and differentiated in serum-containing media (SCM) or XFM (Figure 1, A-C). We found that multiple populations of ECFCs are supported by XFM in the cloning assay (Figure 1D). Immunoprofiling using flow cytometry showed homogenous positive expression of endothelial markers, but cells were negative for mesenchymal or hematopoietic stem cells markers (Figure 1E). To test their capacity for therapeutic angiogenesis, we assessed the in vivo function of XFM-derived ECFCs using the murine OIR model. The OIR model is well-established and mimics the vasoproliferative phase of ocular neovascular diseases such as diabetic retinopathy. In this model, mouse pups are exposed to $75 \%$ oxygen for 5 days, starting at P7 (20). In response to hyperoxia, dramatic vascular remodeling occurs and vessels in the central region of the superficial plexus are obliterated. Upon reexposure to ambient oxygen levels $(\sim 20 \%)$ at P12, the avascular central retina becomes hypoxic; this stimulus drives neovascularization across the superficial vascular plexus (21). To assess vascular regeneration induced by intravitreally injected human cord blood-derived ECFCs, we examined the area of neovascular tuft formation (NV) and vascular obliteration (VO) following OIR. ECFCs were injected into the vitreous body, the semisolid gel structure filling the interior of the eyeball between crystalline lens and retina. Injection of XFM-derived ECFCs at P12 augmented the vascular network significantly to the same extent as SCM-derived ECFCs (Figure 1, F and G). This suggests that ECFCs with XFM can be a potent therapeutic agent for ischemic retinal diseases.

ECFCs provide paracrine support that limits vaso-obliteration during ischemia. To determine the vasoprotective mechanism of ECFCs, we injected GFP-labeled ECFCs into the vitreous to determine their spatial and functional patterns. Consistent with previous findings $(6,7)$, eyes with ECFCs injected at P12 showed significant reductions in NV and VO at P17, the peak of neovascularization (Figure 2, A and B) in the OIR model. This was also confirmed by the injection at P7 (Figure 2, C and D), which resulted in effects comparable to those observed after injection on P12. In order to assess the function of these regenerated retinal vessels, mice were assessed for extravasation of FITC dextran (22). A confocal Z-stack of images rendered in 3 dimensions revealed well-perfused vessels, without any leakage of fluorescent dye in the retinal vessels (Figure 2E). To determine where the exogenously delivered cells localize, we looked for GFP signals within the retinal tissue using confocal microscopy and observed no cells in the retina (Figure $2 \mathrm{~F}$ ). We next imaged the vitreous after flat mounting the posterior lens capsule and retina (Figure $2 \mathrm{G}$ ) and observed a substantial number of GFP-expressing ECFCs in the vitreous body (Figure 2, H-J). This finding strongly suggests that ECFCs provide their rescue through a paracrine effect.

The efficacy of human ECFCS in rescuing OIR using mice lacking various immune-competent cell types. Several aspects of these experiments could elicit an immunologic response, even though the eye is considered to be relatively immune privileged (23). For example, damage-associated molecular patterns that may occur in injected cells may play a role in the induction of innate immunity (24). Immune cells are believed to stimulate or suppress angiogenesis partly by releasing angiogenic/antiangiogenic factors to 
A

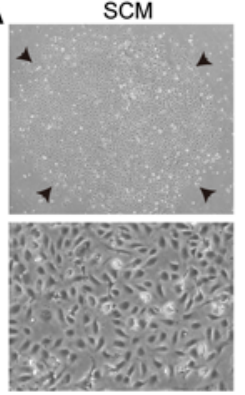

E

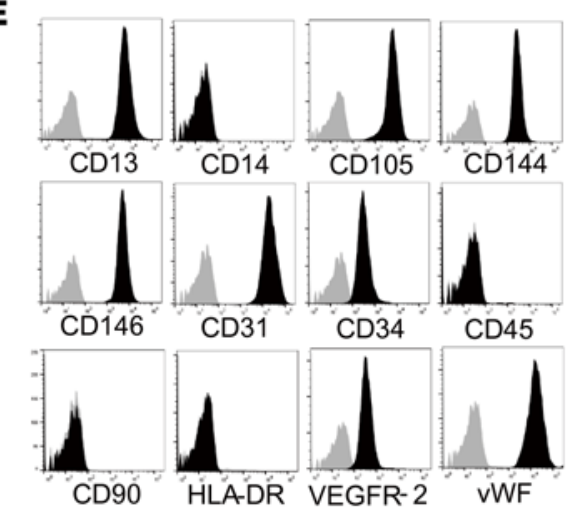

C

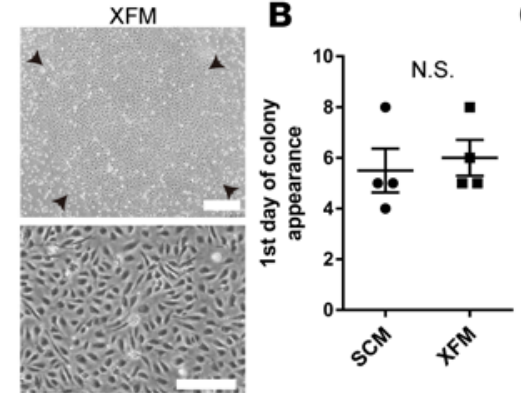

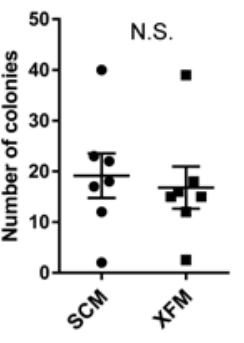

D
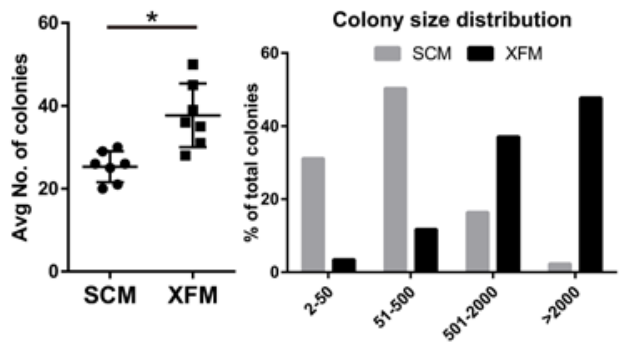
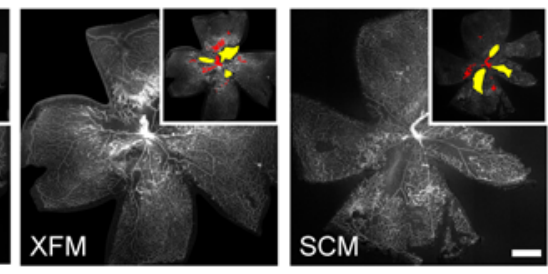

G
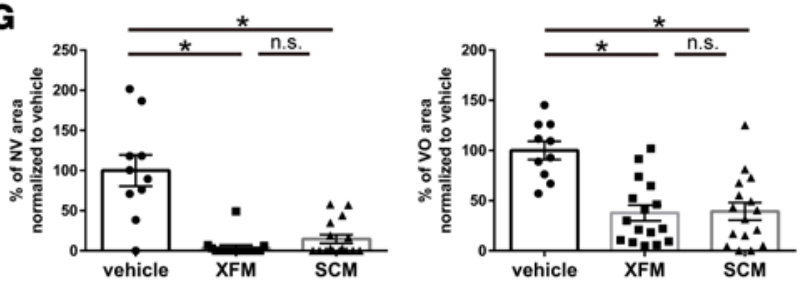

Figure 1. Isolation and amplification of ECFCs using xeno-free media: in vitro and in vivo characterization. (A) Representative photomicrographs of individual human ECFC-derived endothelial cell colonies from umbilical cord blood in serum-containing media (SCM) and xeno-free media (XFM). Morphology of ECFCs derived in XFM did not differ from that of those derived in SCM. Colony of ECFCs derived in XFM at day 10 at low (top row) and high (bottom row) magnification. Scale bar: $500 \mu \mathrm{m}$ (top row); $200 \mu \mathrm{m}$ (bottom row). (B) No significant difference in the mean number of days before initial colony appeared from mononuclear cells after culture initiation in SCM and XFM $(n=4)$. (C) No significant difference in the number of initial EC colonies between culture in SCM and XFM at day $10(n=7)$. (B and C) Error bars represent SEM. (D) Average number of colonies in the cloning assay at day 14 passage 4 ( $P=0.0021$, Student's $t$ test) and distribution of colony size for in vitro cloning assay in SCM and XFM. XFM supports the whole hierarchy of ECFCs. ECFCs derived and amplified in XFM maintained their high proliferative capacity (medium and large colony size) in cloning assays. Error bars represent SD. (E) Phenotypic analysis of ECFCs derived in XFM. Representative flow cytometry histograms of ECFCs derived in XFM show reactivity with EC-expressed marker molecules (right-shifted, black-filled curves compared with gray-filled curves of the appropriate isotype controls) and lack of reactivity with hematopoietic (CD14 and CD45) and mesenchymal stem cell-associated (CD90) markers. (F and G) Rescue of oxygen-induced retinopathy (OIR) by intravitreally injected ECFCs from XFM or SCM, normalized to vehicle injection. (F) Representative CS lectin-stained neural retina flat-mount preparations injected at P12 with vehicle or ECFCs from XFM or SCM. Eyes were isolated from P17 OIR mice. In the insets of F, neovascular tufts (NV) and vaso-obliterated regions (VO) are labeled red and yellow, respectively. Scale bar: $500 \mu \mathrm{m}$. (C) Total area of NV and VO in vehicle and ECFCs from XFM or SCM injected eyes was calculated and plotted ( $n$ $=16$ in ECFCs and $n=10$ in vehicle. ${ }^{*} P<0.001$, Kruskal-Wallis test with Dunn's multiple comparison test). Error bars represent SEM.

endothelial cells (ECs) (25). Therefore, we sought to determine if the rescue effect of human ECFCs in the murine OIR model is a result of an immunologic response. We performed FACS analysis to examine immune cell profiles on OIR retina after ECFC injection with antibodies for myeloid cell marker (CD11b, CX3CR1 and Ly6C\&G) and T cell marker (CD3). As a slight increase of CD11b-positive cells and a decrease of CD3-positive cells were detected (Supplemental Figure 1), we decided to utilize several transgenic mouse lines that lack immune cells to confirm whether immune cell absence affects the effect of ECFC injection on OIR rescue. We used the immunodeficient Rag2 knockout mice, conditional T cell-deficient mice (LCK-Cre(+); R26iDTR/+: LCK-DTR mice), and conditional myeloid-deficient mice (LysM-Cre(+); R26iDTR/+: LysM-DTR mice) (26). In Rag2 knockout mice, significant rescue of both NV and VO was found in the OIR model after intravitreal injection of ECFCs (Supplemental Figure 2A; supplemental material available online with this article; doi:10.1172/jci.insight.89906DS1). In spite of efficient diphtheria toxin-mediated cell depletion in both LysM-DTR and LCK-DTR mice (about 50\% each, Supplemental Figure 3), we detected a significant reparative effect of ECFC injection in both LysM-DTR and LCK-DTR mice mice, which was similar to that in control mice in the OIR model (Supplemental Figure 2, B and C). These results suggest that the rescue effect of ECFCs in OIR is not simply a consequence of immune response associated with such as a cell death. 

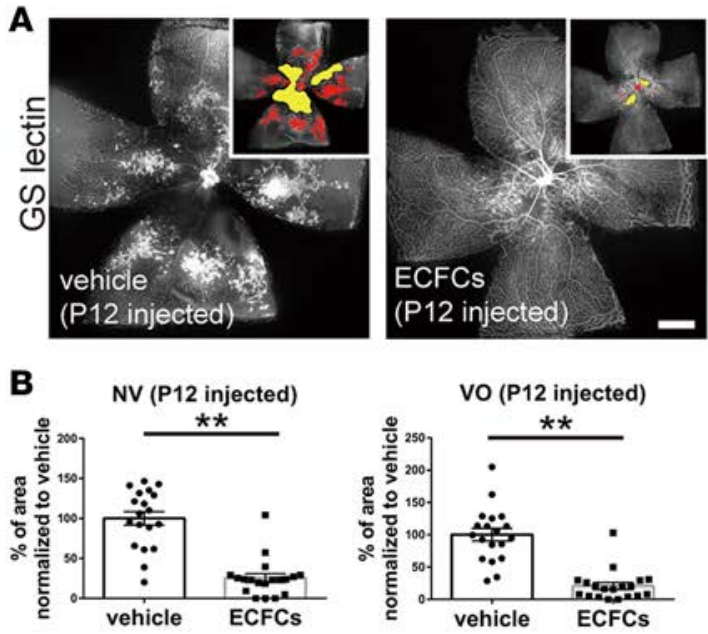

F
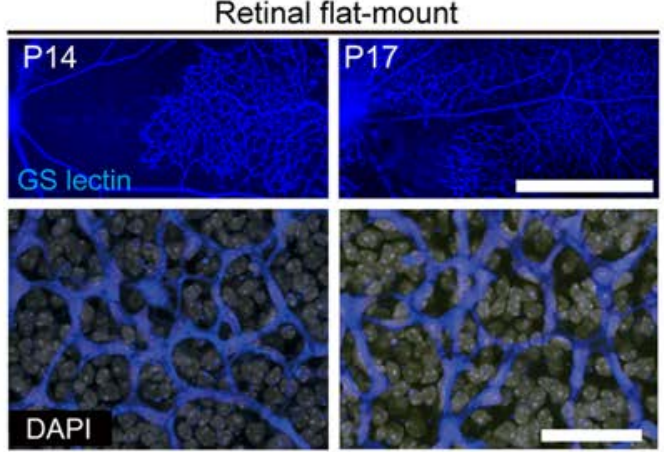

C
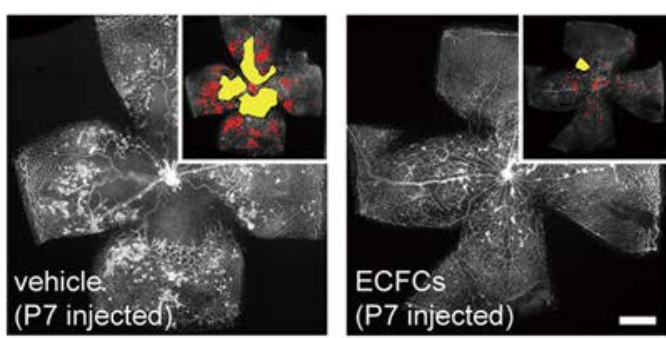

D
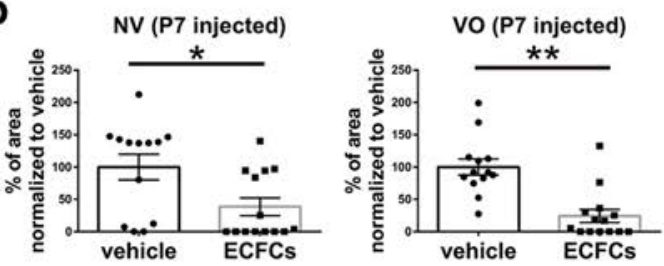

G

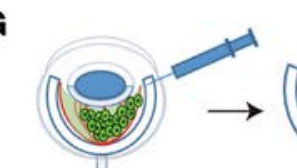

Intravitreal injection

Posterior lens capsule
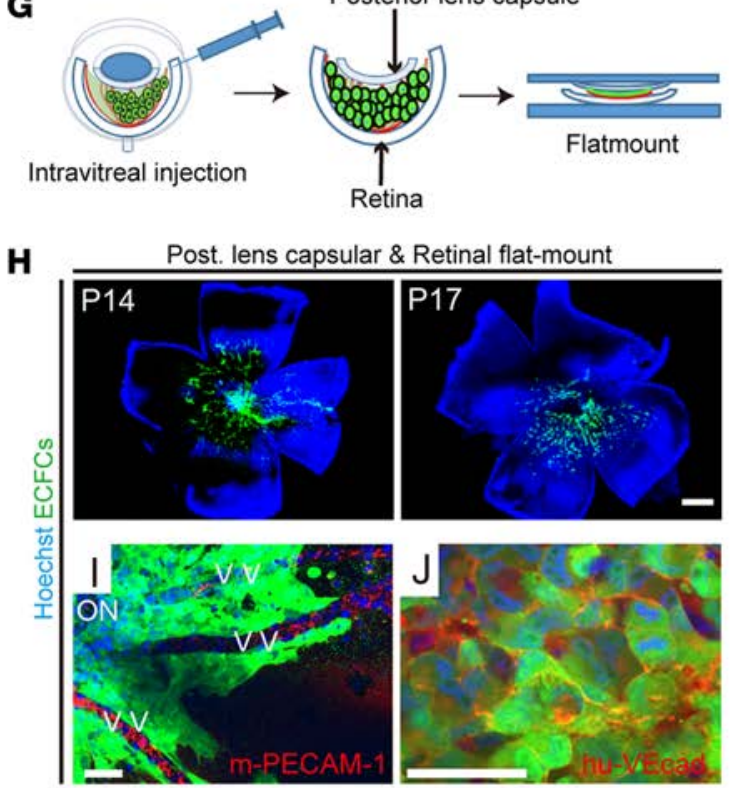
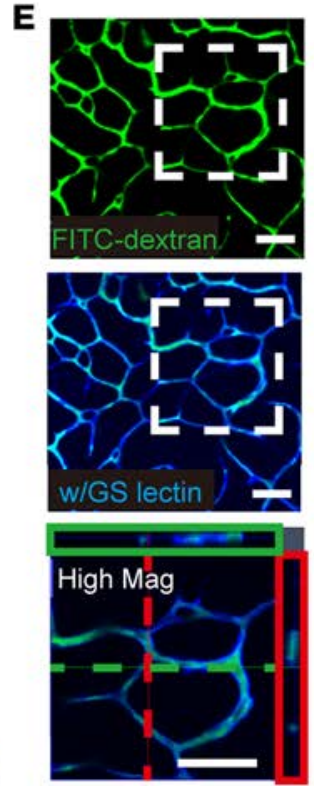

Figure 2. Intravitreally injected human ECFCs regenerate retinal vasculature and localize in the vitreous during oxygen-induced retinopathy. (A-D) Representative GS lectin-stained flat-mount retinas (A and $\mathbf{C}$ ), and quantification (B and $\mathbf{D}$ ), harvested at P17 and treated with human ECFCs or vehicle at P12 (A and B) ( $n=19)$ or at P7 (C and D) ( $n=13$ in vehicle and $n=14$ in ECFCs). In the insets, neovascular tufts (NV) and vaso-obliterated regions (VO) are labeled red and yellow, respectively. (E) FITC dextran perfusion (green) alone and with CS lectin staining (blue) of P12 ECFC-injected retinal vasculature in a oxygen-induced retinopathy model harvested at P17. A high-magnification view of the areas indicated by the white boxes is shown below. Cross-sectional observations of the high-magnification Z-stack images, indicated by the dashed green line (pictured within the green box atop image) and the dashed red line (pictured within the red box adjacent to image) show dextran filling the lectin-stained vessels. (F) Absence of injected GFP-expressing ECFCs (GFP-ECFCs) in flat-mount retinal staining with GS lectin (blue) and/or DAPI (gray). (G) Schematic images of retinal and post-capsular flat-mount mouse eyes. After intravitreal injection of GFP-ECFCs at P12, the cornea, sclera, RPE/choroid, and anterior capsule/nucleus of the lens were removed. (H-J) GFP-ECFCs (green) localized in the vitreous at P14 and P17. (H) Low-magnification images of flat-mount staining of posterior lens capsule and retina and (I and $\mathbf{J})$ high-magnification images with anti-mice PECAM-1 (I, red) and anti-human VE-cadherin antibody staining (J, red). Nuclei were stained with Hoechst33342 (blue). ON, optic nerve; VV, vitreous vessel. Scale bar: $25 \mu \mathrm{m}(\mathbf{E}) ; 50 \mu \mathrm{m}$ (F [bottom], I, and J); $500 \mu \mathrm{m}(\mathbf{A}, \mathbf{C}, \mathbf{F}$ [top], and $\mathbf{H}) .{ }^{*} P<0.05,{ }^{*} P<$ 0.001 , Mann-Whitney test. Error bars represent SEM.

A subset of ECFCS with high CD44 expression levels were more effective at rescuing the OIR phenotype and retinal degeneration. We confirmed that in general ECFCs defined by Yoder et al. $(3,27)$ display homogenous clonal characteristics (Supplemental Figure 4), but we did determine that CD44 levels were markedly heterogeneous (Supplemental Figure 5). The percentage of CD $44^{\text {hi }}$ ECFCs was $47.5 \% \pm 23.6 \%$ and CD $44^{\text {lo }}$ ECFCs was $23.1 \% \pm 4.95 \%$. We then tested if CD44 expression correlated with vasoprotective potential. Indeed CD $44^{\text {hi }}$ ECFCs prevented vaso-obliteration in the OIR model more effectively than CD $44^{\text {lo }}$ ECFCs (Figure 3, A-C). To confirm that the level of CD44 expression influences the rescue capacity in OIR mice, 
we generated CD44 knockdown ECFCs using shRNA (Supplemental Figure 6). Knockdown of CD44 dramatically reduced the rescue potential (Figure 3, D and E). Not only did the CD $44^{\text {hi }}$ ECFCs prevent vaso-obliteration better than $\mathrm{CD} 44^{\text {lo }}$ cells, they also induced quiescence of ECs and prevented excessive permeability typically associated with the OIR phenotype (Supplemental Figure 7, A and B). To investigate if ECFCs also exert neuroprotective effects, we evaluated the inner retina that is affected during OIR. The inner plexiform layer (IPL) and inner nuclear layer (INL) showed reduced thickness in OIR eyes, but those were not reduced at P30, when CD44hi ECFCs were injected at P12 (Figure 3, F and G). Accordingly, for retinal function analysis, we performed electroretinography (ERG) experiments at P30 for eyes received ECFCs at P12. Scotopic ERG $b$ wave analysis demonstrated that injection of CD44 $4^{\text {lo }}$ ECFCs improved inner retinal function, which is disturbed in OIR eyes (Supplemental Figure 8).

We also injected ECFCs in mice with inherited retinal degeneration. The retinal degeneration10 (rd10) mouse line $\left(P d e 6 b^{\text {rd10/rd10 }}\right)$ shows a relatively slow progression of photoreceptor cell death, beginning around P21. The ONL degenerates, and cells die mainly by apoptosis, such that ONL thickness drops abruptly to two to three layers of nuclei between P20 and P25; only a single layer of nuclei remains by P45 (28-30). We decided to inject ECFCs or vehicle control at P14 and evaluated neurovascular degeneration thereafter. Flat-mount staining with isolectin B4 at P32 was used to evaluate the deep layer of the retinal vasculature, which starts to degenerate shortly after the onset of photoreceptor cell death. Retinal vessels were significantly preserved in eyes injected with CD $44^{\text {hi }}$ ECFCs compared with eyes injected with CD $44^{\text {lo }}$ ECFCs or vehicle alone (Figure 4, A and B, and Supplemental Figure 9A). For evaluation of neuroprotection, we measured ONL thickness at P28. Eyes injected with CD44hi ECFCs had a significantly thicker ONL layer, with expression of several cone- and rod-specific markers (Figure 4, C-E, and Supplemental Figure 9B). However, the effect of ECFC injection was transient and could not be detected in 2-month-old mice (Supplemental Figure 9). The trophic effect of injected CD $44^{\text {hi }}$ ECFCs was confirmed by TUNEL staining at P21, at which time fewer apoptotic cells were observed (Figure 4, F and G). Functional analyses using full-field ERG for the integrity of the photoreceptors, the second- and third-order neurons in either dark-adapted (scotopic) or light-adapted (photopic) animals, were performed. ERG revealed a significant preservation of both scotopic (rod-driven) and photopic (cone-driven) responses in CD $44^{\text {hi }}$ ECFC injected eyes (Figure 5, A and B). Collectively, angiocine factors released by ECFCs potentiate angiogenic repair as well as neurotrophic rescue in an inherited murine retinal disease model. To evaluate the mechanism of neurotrophic rescue by ECFCs, we screened for expression of human neurotrophin genes expressed in injected ECFCs-shCD44 on RD10 mice. In a qPCR array for 84 neurotrophin and receptor genes, there were no significant reductions in gene expression in the CD44 knockdown cells isolated from RD10 mice 2 days after injection (our unpublished observations). Based on this observation, we hypothesized that an indirect mechanism may be responsible for the neurotrophic activity of the ECFCs. A qPCR-based gene profile with 84 probes for mouse neurotrophin and receptor genes that contribute to neuronal cell growth, differentiation, and regeneration was performed. At P22, RNA was extracted from RD10 retina which was injected with ECFCs at P14 and gene profiling demonstrated that eyes received CD $44^{\text {hi }}$ ECFCs exhibited upregulation of 12 mouse neurotrophin-related genes compared with CD44 ${ }^{\text {lo }}$ ECFCs (Figure 5C). Function of those genes included cellular protection for stress $(\mathrm{Bcl} 2, \mathrm{Crh}$, and $\mathrm{Hspb} 1)$ and transcription factors (Stat1 and Stat2). Those data suggest that expression of vasculotrophin in injected ECFCs, but not neurotrophin, potentiates photoreceptor preservation in RD10 mice.

Characterization of intravitreally injected ECFCs. To determine the mechanisms that mediate the vasculotrophic effects of ECFCs, we set out to examine if CD44 ${ }^{\text {hi }}$ cells exhibited higher affinity for the vitreous and/or if they had increased intracellular signaling. CD44 is a known adhesion molecule that binds HA (which is highly enriched in the vitreous) (13). We examined flat-mounted retinas and vitreous samples of OIR mice after intravitreal injection of GFP-labeled conventional ECFCs and CD44 knockdown ECFCs. Almost the same amount number of ECFCs were detected in both groups of control and CD44 knockdown ECFCs in flat mounts (Figure 6, A-D) and in cross-sectioned retinas (Figure 6, E-H). Using FACS and histological analyses of P14 retinas (Figure 6, I and J), we did not detect any significant difference in cell number between control and knockdown GFP-labeled cells. These results suggest that the enhanced rescue effect of CD44 ${ }^{\text {hi }}$ cells in OIR mice is not due to enhanced adhesion.

High CD44 levels can enhance some intracellular cascades, since, upon activation, the receptor is cleaved and released into the cytoplasm. To determine how CD44 receptor density (and their microenvironments) affect gene expression in ECFCs, we performed qPCR-based gene profiling on ECFCs extracted 
A
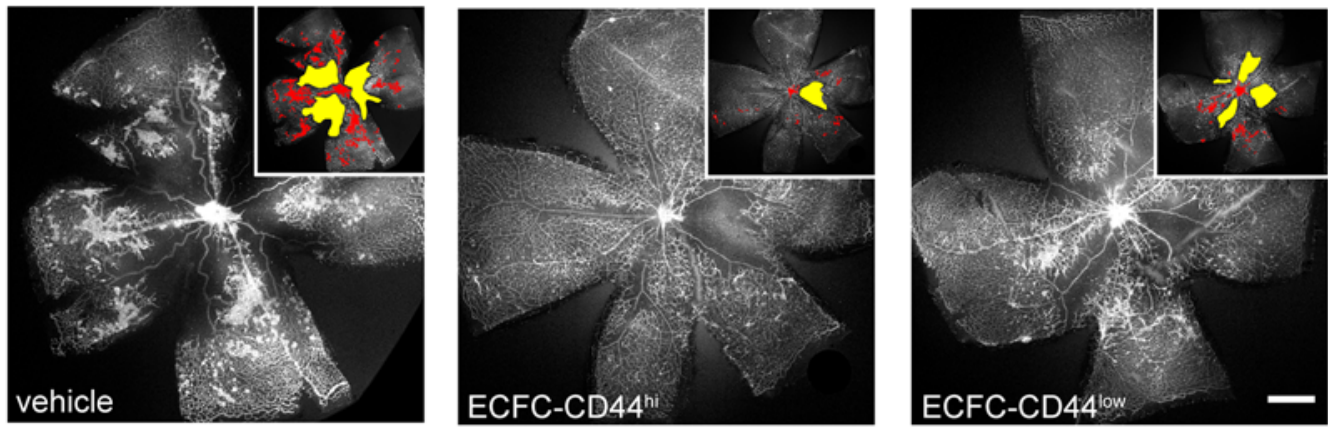

B

G44-26

DB105

D

shCD44
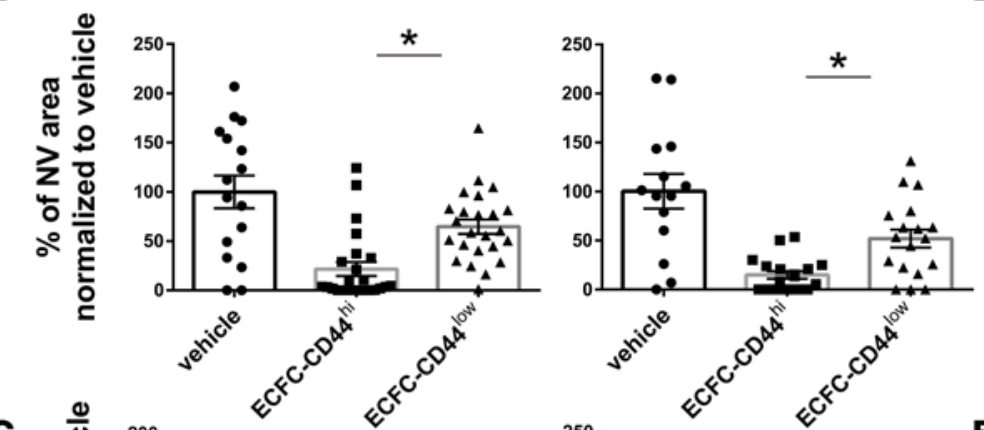

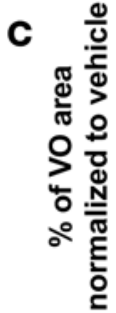
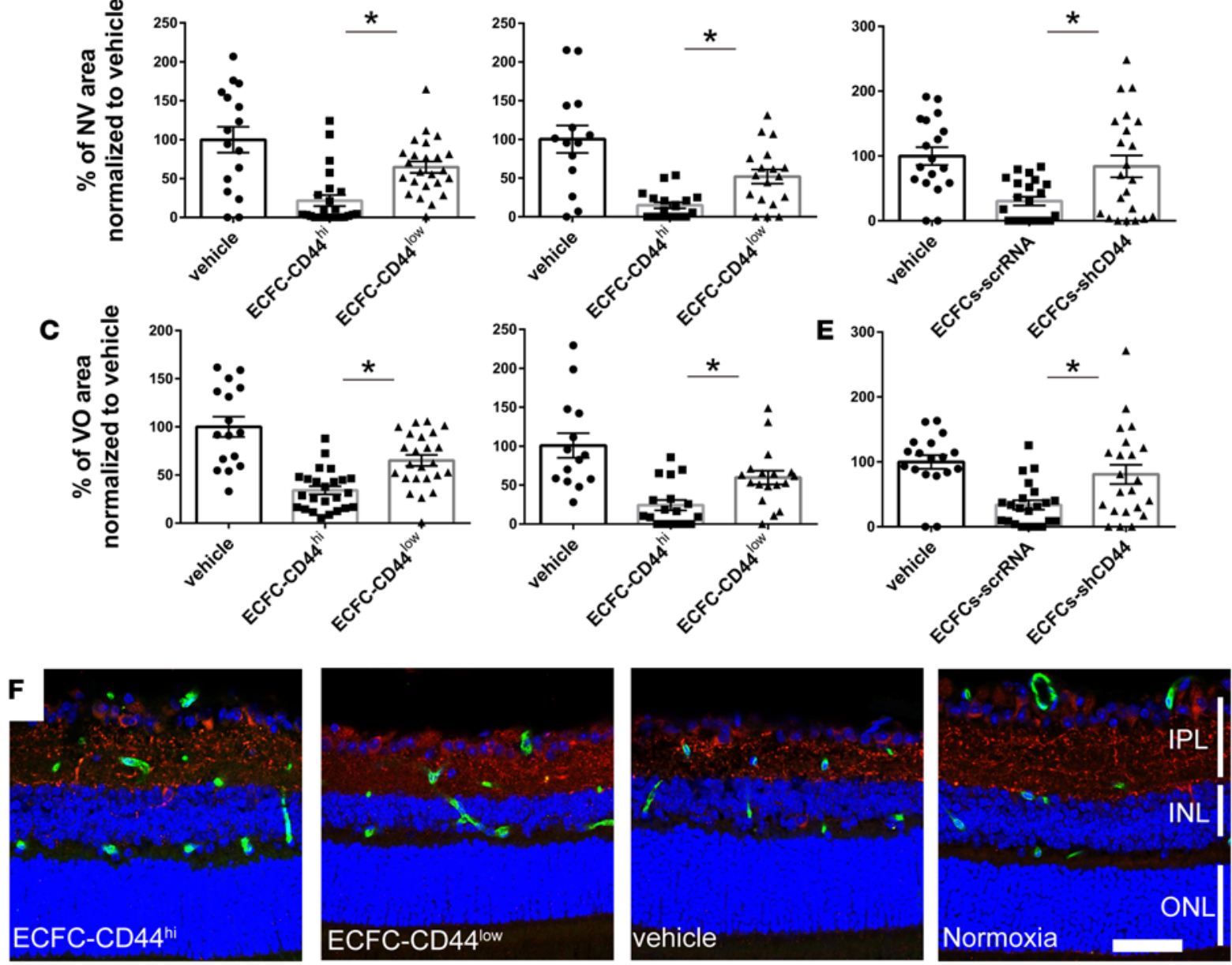

G

IPL

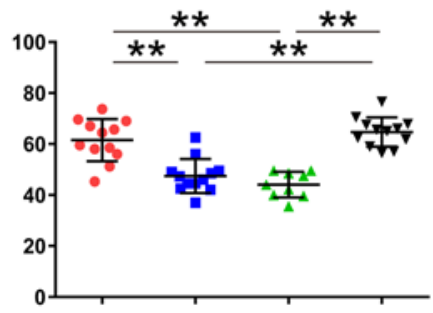

INL
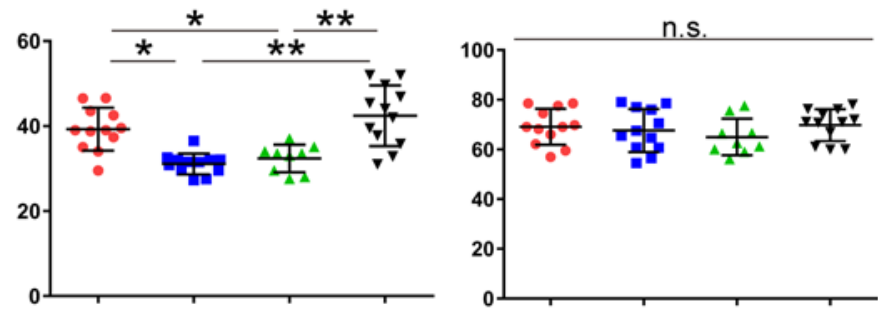

- ECFC-CD44 ${ }^{\mathrm{hi}}$

- ECFC-CD44 ${ }^{\mathrm{low}}$

$\Delta$ vehicle

v Normoxia 
Figure 3. Subpopulation of ECFCs facilitates regeneration of retinal vasculature and reduces pathological angiogenesis in oxygen-induced retinopathy model. (A) Representative GS lectin-stained flat-mount retinas. Scale bar: $500 \mu \mathrm{m}$. (B and C) Oxygen-induced retinopathy (OIR) quantification for eyes injected at P12 with CD44 ${ }^{\text {hi }}$ ECFCs, CD44 ${ }^{\circ}$ ECFCs, or vehicle. Retinas were harvested at P17 using a different clone of CD44 antibody. Results for neovascular tufts (NV) are shown in B and vaso-obliterated regions (VO) are shown in C. Quantifications show the results of each clone of antibody (G44-26 or DB105) for CD44 selection. ${ }^{*} P<0.01$, Kruskal-Wallis test with Dunn's multiple comparison test; C44-26, $n=24$ in ECFCs and 16 in vehicle; DB105, $n=18$ in ECFCs and 14 in vehicle. ( $\mathbf{D}$ and $\mathbf{E}$ ) Quantification of OIR retinas, harvested at P17, that were injected at P12 with control knockdown (KD) ECFCs (ECFCs-scrRNA), CD44-KD ECFCs (ECFCs-shCD44), or vehicle. Results for NV are shown in $\mathbf{D}$, and results for VO are shown in $\mathbf{E}$. ${ }^{*} P<0.01, n=22$ in ECFCs and 18 in vehicle. (F and $\left.\mathbf{G}\right)$ Retinal sections at P30, after injection at P12, show highly efficient neuroprotection with CD44 ${ }^{\text {hi }}$ ECFC injection in the OIR model. (F) Results of immunohistochemistry with MAP2 (red) and PECAM-1 (green) antibodies for OIR retinas injected with CD44 ${ }^{\text {hi }}$ ECFCs, CD44 $4^{10}$ ECFCs, or vehicle, compared with retinas from mice housed in normoxic conditions. Nuclear staining was performed with Hoechst33342 (blue). IPL, inner plexiform layer; INL, inner nuclear layer; OPL, outer plexiform layer. Scale bar: $50 \mu \mathrm{m}$. (C) Quantification for each retinal layer thickness of central retina after ECFC injection. $n=12$ in ECFCs and normoxia, $n=9$ in vehicle. ${ }^{*} P<0.01,{ }^{* *} P<0.001$. 1-way ANOVA with Tukey analysis. Error bars represent SEM in B-E and SD in $\mathbf{G}$.

from the vitreous of murine retinas after injection at P13 (Figure 6, I and J). Several proangiogenic factors were found to be downregulated in ECFCs-shCD44-GFP compared with ECFCs-scrRNA-GFP (Figure $6 \mathrm{~K})$, strongly suggesting that reducing CD44 levels in ECFCs affects the degree of downstream gene activation. These data also suggest that high levels of CD44 correlate with higher vascular rescue due to the improved ability to respond to microenvironmental cues and activate gene expression.

$E C F C$-conditioned media facilitate vascular repair in OIR. The observation that ECFCs home to the lens and vitreous confirms suggestions made by others that ECFCs function largely in a paracrine manner $(10,11)$. To confirm this, we analyzed gene expression for 84 angiogenic growth factors in $\mathrm{CD} 44^{\text {hi }}$ and CD $44^{\text {lo }}$ ECFCs grown in monolayer culture. Surprisingly, we did not detect upregulation of any genes in CD44 4 hi ECFCs (Supplemental Figure 7). Since gene expression can be microenvironment dependent, we decided to use a 3D collagen-based cell culture system to mimic the in vivo conditions of vitreous (Figure 7A). 3D culture appears to alter the ECFC gene profile, based on qPCR experiments comparing ECFCs cultured in two versus three dimensions. Specifically, CD44 knockdown ECFCs grown in 3D gel culture downregulated several proangiogenic factors compared with control ECFCs, though this difference was not observed in 2D culture (Figure 7B and Supplemental Figure $10 \mathrm{~A})$. We confirmed a similar gene expression pattern in CD44 $4^{\text {hi }}$ and CD $44^{\text {lo }}$ ECFCs cultured in 3D gel culture compared with that in CD44 knockdown ECFCs (Supplemental Figure 10B). We assayed conditioned media collected from 3D culture of ECFCs with a multipanel ELISA, which detected the relative expression level of 55 angiogenesis-related proteins (Figure 7C). Together with absolute quantification done by regular ELISA (Figure 7D), we detected downregulation of IGFBP2 and IGFBP3 in conditioned media from ECFCs-shCD44-GFP cultured in 3D, compared with ECFCs. Moreover, we measured human IGFBP2 and IGFBP3 protein levels in mouse OIR eyes after injection of ECFCs using Meso Scale analysis based on electrochemiluminescence detection technology. Significant reductions of both human IGFBP2 and IGFBP3 were detected in OIR eyes that received ECFCs-shCD44 compared with control ECFCs (Figure 6L).

To obviate the need to sort and utilize only $\mathrm{CD} 44^{\text {hi }}$ cells, or to use transgenic approaches to overexpress CD44 in CD44 cells, we tested if conditioned media from ECFCs might rescue the OIR phenotype. Indeed, conditioned media from control ECFCs reduced NV and VO more effectively than conditioned media from CD44 knockdown ECFCs or media alone (Figure 7, E and F). In addition, since IGFBP2 and IGFBP3 and CD44 expression levels are correlated in ECFCs, we injected recombinant human protein of IGFBP2/3; this also rescued the OIR phenotype (Figure 8, A-D). Moreover, the rescue effect of IGFBPs secreted from ECFCs in OIR was cancelled by depleting IGFBP2 and IGFBP3 in the supernatant of ECFCs using neutralizing antibodies with protein $\mathrm{G}$ magnetic beads (Figure 8, E-G). Rescue effect for NV was impaired when IGFBP2 was depleted, and moreover, the effect for VO was cancelled when both IGFBPs were depleted. Thus, IGFBP2 and IGFBP3 are critical cues in the trophic mechanism of ECFC-based therapy for neurovascular diseases.

\section{Discussion}

In cell therapy for general regenerative medicine, it is well-known that transplanted cells often provide substantial benefit to the recipient through transdifferentiation and functional replacement of damaged cells as well as through paracrine effects (31). Evidence for this in ECFC biology comes from the relatively low number of cells that engraft at the ischemic site, which nonetheless can provide a substantial therapeutic benefit $(6,8)$. Furthermore, it is rare to find vessels that consist of injected cells only. The purpose of this study was to determine if human ECFCs exert trophic effects of angiogenesis that can mitigate pathological consequences of retinal disease, including vascular damage and neural cell loss. 
A

ECFC-CD44

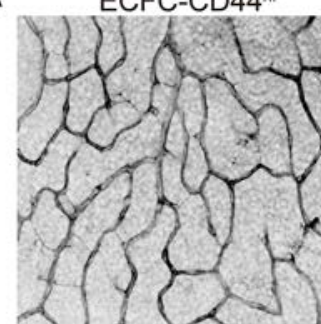

C

ECFC-CD44 ${ }^{\text {hi }}$

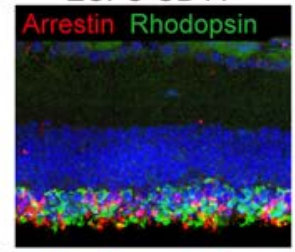

D

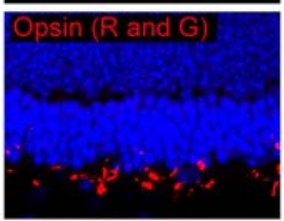

$\mathbf{F}$

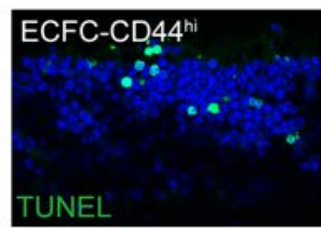

ECFC-CD44

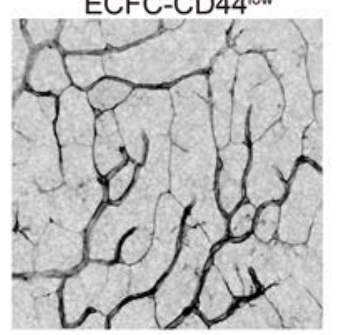

ECFC-CD44
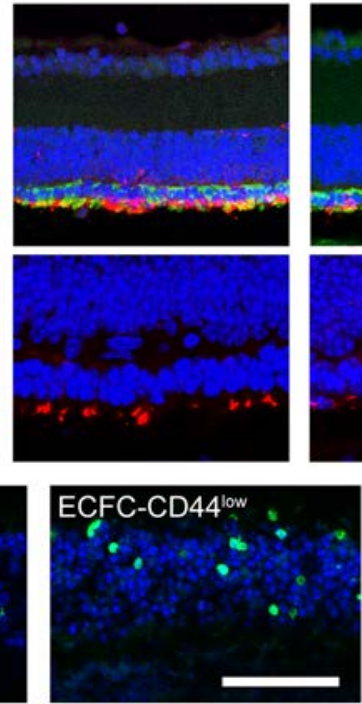

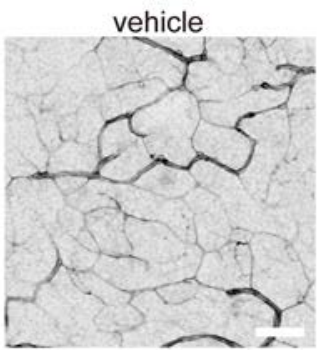

vehicle

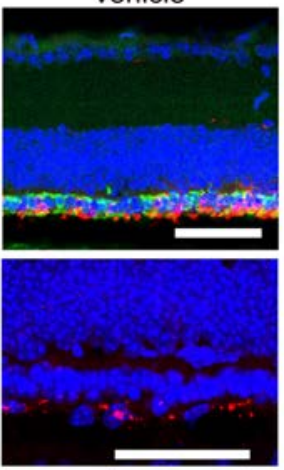

G

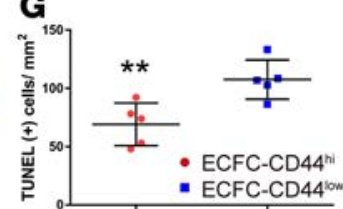

B Pde6b rdtardio

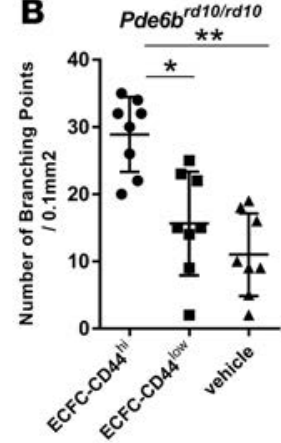

E
E ONL thickness (um)

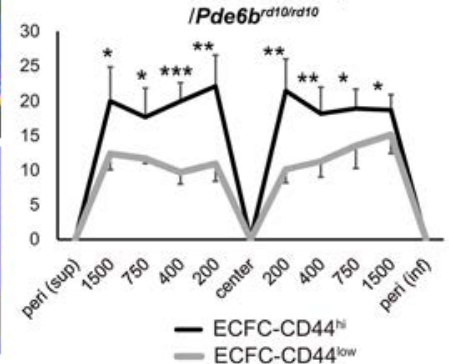

Figure 4. ECFCs promote transient neurovascular protection in inherited retinal degeneration mice. ( $A$ and B) Representative images (A) and quantification (B) of staining for deep plexus of retinal vasculature with GS lectin on P32 RD10 retinas show rescue of vascular atrophy with CD44 ${ }^{\text {hi }}$ ECFCs. $n=8$. (C and D) Immunohistochemistry of retinal sections harvested at P28 after injection on P14 in RD10 mice shows a highly efficient neuroprotective role of CD44hi ECFCs. (C) Red, arrestin; green, rhodopsin; blue, Hoechst33342. (D) Red, opsin (red/green); blue, Hoechst33342. (E) Quantification for outer nuclear layer thickness. $n=5$. (F and $\mathbf{G})$ TUNEL staining on P21 RD10 mouse retinas shows fewer apoptotic cells in CD44 hi ECFCinjected eyes. (G) Quantification of TUNEL staining in $\mathbf{F}$. $n=5 .{ }^{*} P<0.05,{ }^{* *} P<0.01$, Student's $t$ test. Scale bar: 50 $\mu \mathrm{m}$. Error bars represent SD.

We present several lines of evidence showing that a population of ECFCs, which is defined by CD44 expression, can promote vascular and neuronal protection in the murine OIR model and RD10 mice through the secretion of IGFBPs. ECFCs expressing high levels of CD44 had a trophic effect on the neurovasculature in OIR and RD10. Using a flat-mount technique for both posterior capsule of lens and retina, we observed injected ECFCs localized to the vitreous. Purifying injected cells enabled us to analyze the angiogenic genes modulated by CD44 signaling. Second, conditioned media from 3D cell culture could promote a reparative effect in OIR. Proteomic analysis focusing on angiogenic factors in condition media revealed the presence of IGFBP2 and IGFBP3. Third, IGFBP2 and IGFBP3 secreted from ECFCs could rescue OIR model. Exogenous administration of human recombinant IGFBP2 $(32,33)$ or IGFBP3 $(34,35)$ protein rescued the OIR phenotype.

IGFBPs were originally characterized as reservoirs of circulating IGFs, but they are now understood to possess many IGF-independent functions (36). IGFBPs, which reportedly localize in the pericellular and intracellular compartments, regulate cell growth and survival (36). As expected in our study, intravitreal injection of human recombinant IGFBP2 and IGFBP3 promoted a reparative effect in OIR. IGFBP2 has been reported to interact with integrin $\alpha_{v} \beta 3$ (37) and $\alpha_{5} \beta 3$ (38) during angiogenesis. Recent extensive analyses for regenerative factors secreted from ECs revealed IGFBP2 as one of the candidate angiocrine factors $(39,40)$. IGFBP3 has been shown to regulate vascular repair in the OIR model $(34,35)$.

We showed that ECFCs promote neurovascular protection in both OIR and inherited retinal degeneration mouse models. ECFCs restore the thickness of inner retina in the OIR model. Interestingly, ECFCs exhibited a rescue effect on the thickness of ONL in the RD10 mouse model. In general at P17 in OIR model, vascular development has not been accomplished at the level of deep vasculature which supply blood to ONL so ischemic lesion is possibly limited to inner retinal layer. On the other hand, inherited photoreceptor degeneration occurs at the level of the outer retina. One of causative reasons for photoreceptor loss has been concluded to be oxidative stress (41). Because a number of studies have shown IGFBPs' role for exerting a pleiotropic antioxidant effect (42), we speculate that the paracrine secretion from ECFCs could restore the vasculature and neurons from different locations in different models.

Currently, several clinical trials of stem cell therapy for retinal neurovascular diseases, including AMD, RP, and diabetic retinopathy, are ongoing (43). Almost half of those trials are using cells of mesodermal origin, 
A
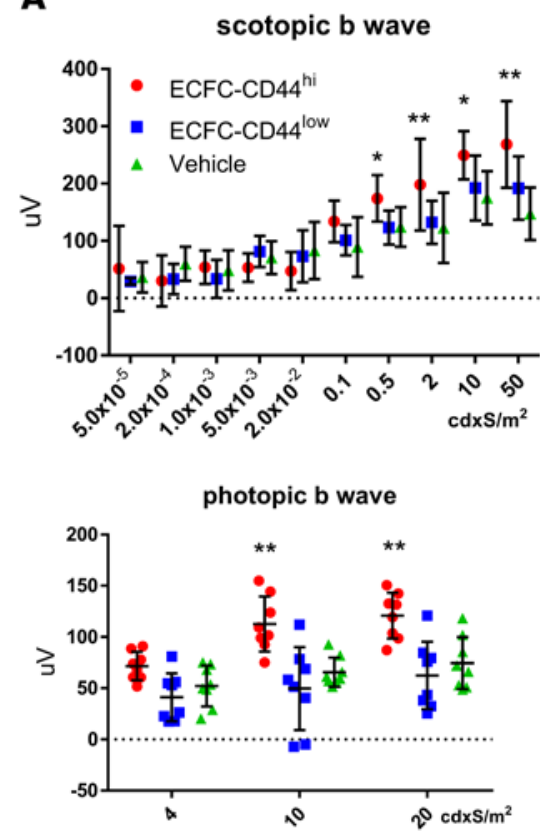

B

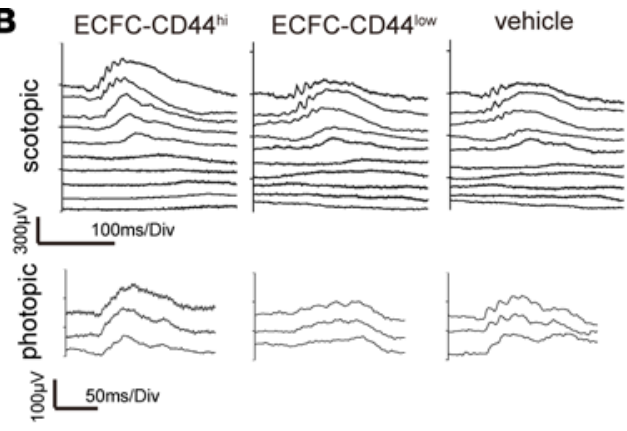

C

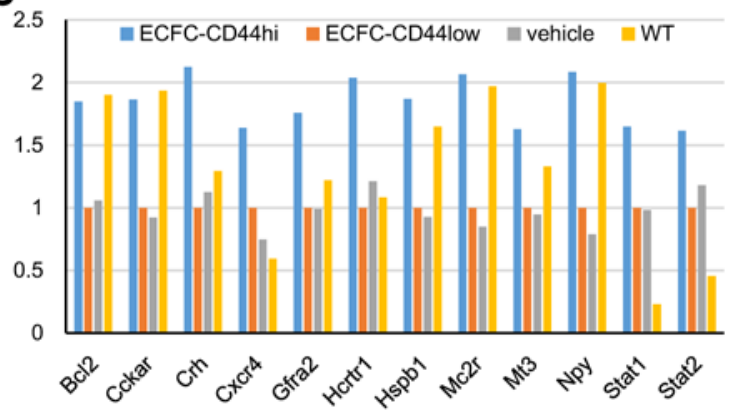

Figure 5. ECFCs promote functional rescue in inherited retinal degeneration mice. (A and $B$ ) ERG recordings to measure the function of retinas at P28 injected with CD44 hi ECFCs or control cells (CD44 ${ }^{10}$ ECFCs) or vehicle at P14. Retinal function was rescued by CD44hi ECFC injection. Error bars represent SD. (C) Relative mRNA expression values from qPCR array gene-profiling analysis of 84 mouse neurotrophin- and receptor-related genes expressed on RD10 or WT (noninjected) whole retinas at P22 after injection of CD44 hi ECFCs, CD44 ${ }^{10} E C F C s$, or vehicle at P14. $n=3-4$. Genes dysregulated by $>1.5$-fold with $P$ values of less than 0.05 were plotted. ${ }^{*} P<0.05$, ${ }^{*} P<0.01,1$-way ANOVA with Tukey analysis.

namely, bone marrow-derived mesenchymal stem cells. Similar to our experiments, those cells were delivered with intravitreal injection. EPCs, which are also of mesodermal origin, are recognized as consisting of two cell populations; early EPC and ECFCs. The latter are also referred as outgrowth endothelial cells $(27,44)$. Compared with early EPCs, ECFCs had been thought to secrete fewer trophic factors (44). In agreement with these reports, we did not detect upregulation of VEGF and HGF in supernatant from $3 \mathrm{D}$ culture of ECFCs. These data suggest that ECFCs did not release those growth factors for their own survival. However, when in $3 \mathrm{D}$ gel culture or the vitreal microenvironment, ECFCs were stimulated to secrete trophic factors such as IGFBPs.

This study focused on angiogenesis-related genes or proteins in our -omics analy-

sis (Figures 4-6 and Supplemental Figures 6 and 7). Nevertheless, other factors released from ECFCs should also be considered. For example, inflammatory cytokines are increasingly recognized as playing an important role in tissue regeneration (45). One group demonstrated that EPC-derived CCL2 recruited neuroprotective microglia in an acute retinal degeneration model (46). Interaction between HA and CD44 has known to induce chemokine expression, which recruits and activates leukocytes (47).

Compared with other reports demonstrating the enhancement of angiogenesis using ECFCs, our study did not detect the homing of cells to the ischemic lesion. Rather, intravitreally injected ECFCs remained in the vitreous. For clinical application, using conditioned media or IGFBP-based therapy would also be a possible alternative strategy. Furthermore, using XFM media, in which ECFCscan be derived, will be important in the development of cell-based therapies using these cells. In addition, it may be possible to transfect these cells to overexpress IGFBPs or other bioactive molecules, semipermeably encapsulate the cells near the site of neurovascular degeneration, and, thus, create a microenvironment supportive of vascular healing and neuronal maintenance.

\section{Methods}

Animals. OIR was induced in C57BL/6J mice according to the protocol described in ref. 20. Briefly, P7 pups and their mothers were transferred from room air to an environment of $75 \%$ oxygen for 5 days and afterward were returned to room air. The hyperoxic environment was created and maintained using a chamber from Bio-Spherix. Under these conditions, large hypovascular areas formed in the central retina during hyperoxia in $\mathrm{C} 57 \mathrm{BL} / 6 \mathrm{~J}$ mice, and abnormal preretinal neovascularization occurred after return to normoxia, peaking at around P17 and ultimately resolving. For immunocompromised mice, we used Rag2 knockout mice that were on a C57BL6 background, because we reported previously that we could induce OIR in C57BL6J wild-type mice but not in the BALB/cByJ strain (18). In addition, we crossed LCK ( $\mathrm{T}$ cell marker) Cre mice (Cg-Tg(Lck-cre)548Jxm/J; The Jackson Laboratory [JAX]) or LysM (myeloid cell marker) Cre mice (Lyz2tm1(cre)Ifo/J; JAX) with a mouse strain in which the human diphtheria toxin receptor (iDTR) is knocked into the ROSA26 

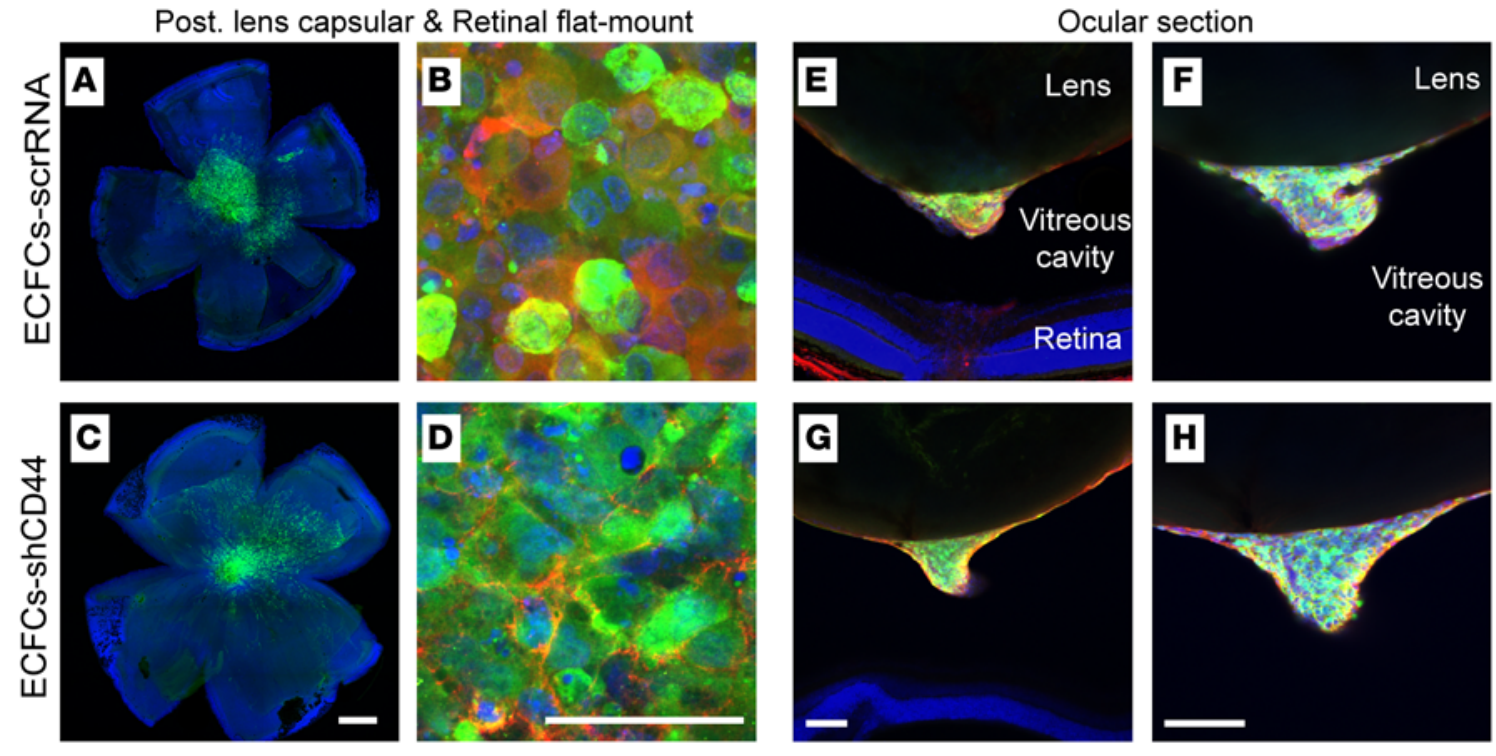

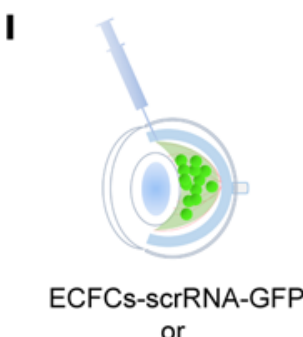

ECFCs-shCD44-GFP

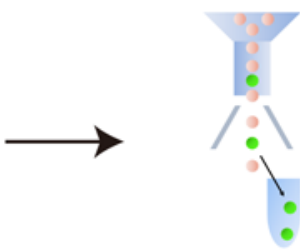

Isolation GFP+ cells from eyes

Gene expression analysis

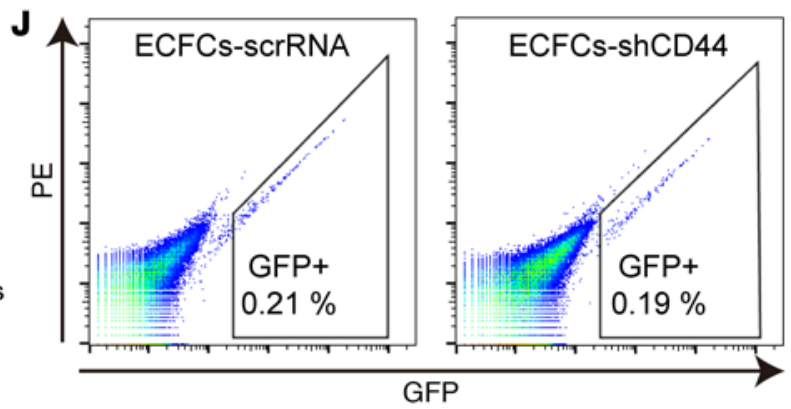

$\mathbf{K}$

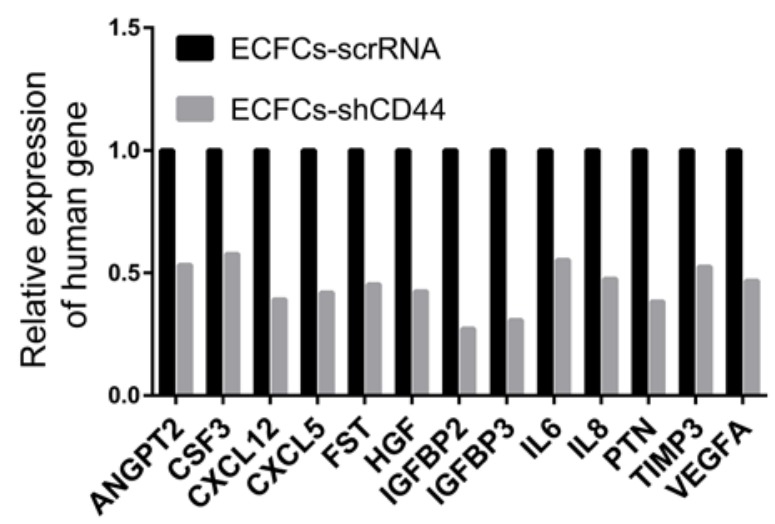

$\mathbf{L}$

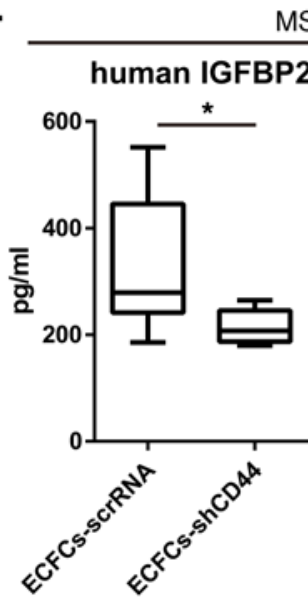

MSD analysis

human IGFBP3

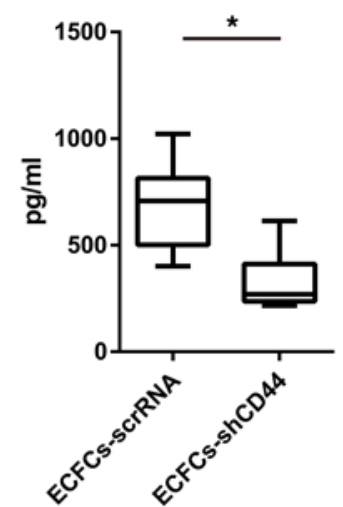

Figure 6. Morphological and molecular characterization of ECFCs in the vitreous regulated by CD44. (A-H) OIR eyes after injection of CD44 knockdown ECFCs did not show apparent morphological differences in samples of flat-mount stained retinas (A-D) or eye sections (E-H). Green, GFP; red, human-VE-cadherin; blue, Hoechst33342. (A-D) Low-magnification (A and $\mathbf{C}$ ) or high-magnification (B and $\mathbf{D}$ ) images of flat-mount staining for posterior lens capsule and retina harvested at P14 after injection at P12 of control ECFCs (ECFCs-scrRNA) (A and B) or CD44 knockdown ECFCs (ECFCs-shCD44) (C and $\mathbf{D})$. (E-H) Low-magnification (E and $\mathbf{G}$ ) or high-magnification images ( $\mathbf{F}$ and $\mathbf{H})$ of immunohistochemistry for eyeball sections harvested at $\mathbf{P 1 4}$ after injection at P12 of ECFCs-scrRNA (E and F) or ECFCs-shCD44 (G and H). Scale bar: $500 \mu \mathrm{m}$ (A and C); $50 \mu \mathrm{m}$ (B and D); $100 \mu \mathrm{m}$ (E-H). (I) Experimental schema for isolation of P12 injected ECFCs using FAC sorting at P13. (J) Flow cytometry analysis for GFP-positive ECFCs-scrRNA or ECFCs-shCD44 from OIR eyes. (K) qPCR-based gene profile analysis for angiogenic growth factors expressed on injected ECFCs-scrRNA or ECFCs-shCD44. Cenes dysregulated by $>1.5$-fold with $P$ values of less than 0.05 were plotted. (L) Mesoscale discovery (MSD) analysis based on electrochemiluminescence detection technology for human IGFBP2 and IGFBP3 protein levels in a whole OIR eyes at P14 injected ECFCs at P12. Error bars represent Min to Max. $n=4$. ${ }^{*} P<0.05$, Mann-Whitney test. 
A

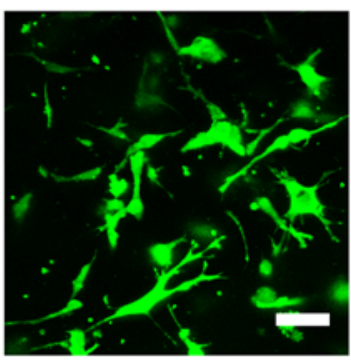

B

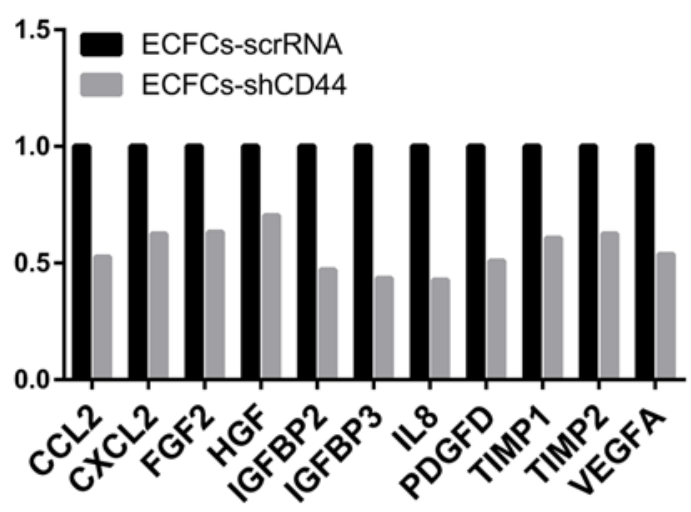

C
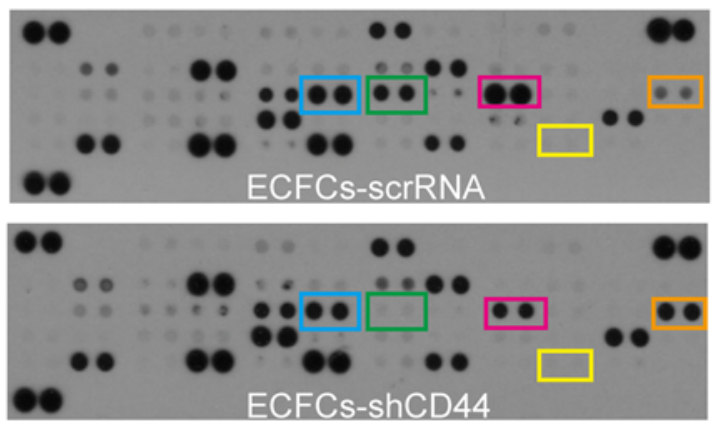

D

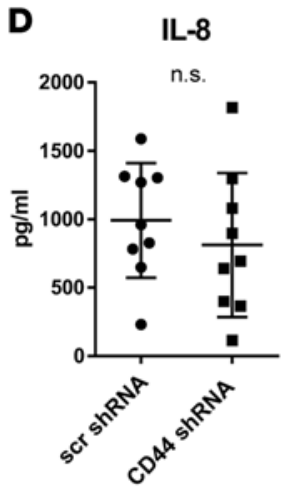

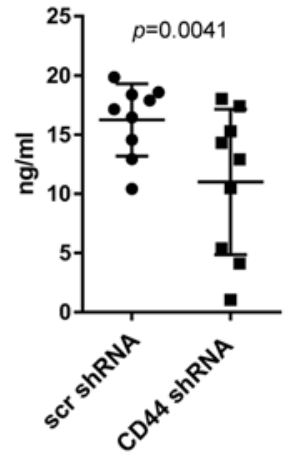

IGFBP2
IGFBP3

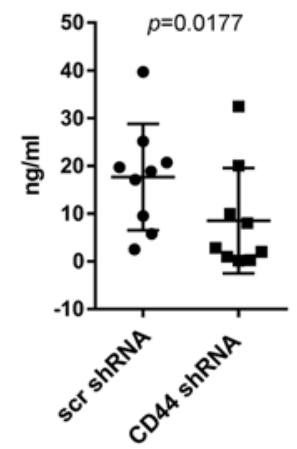

E
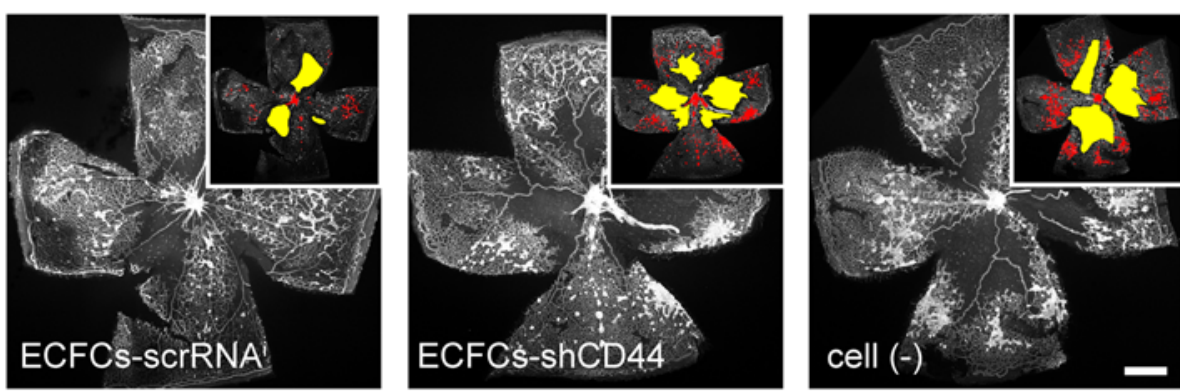

$\mathbf{F}$

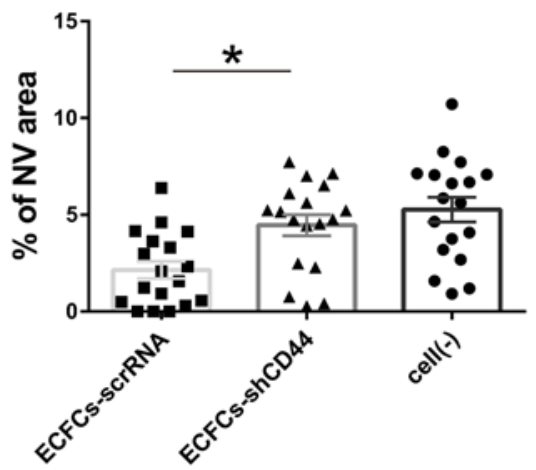

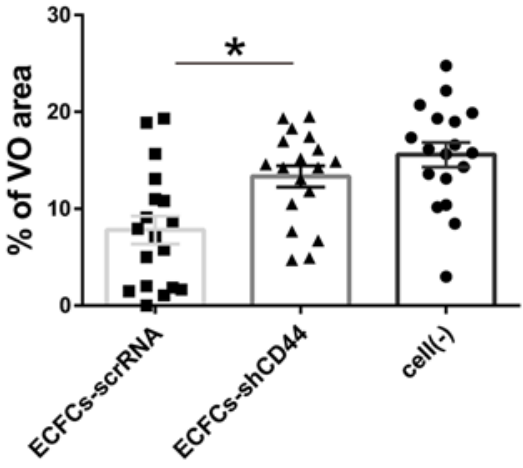

Figure 7. Angiocrine factors secreted in cell-free media from 3D gel culture of ECFCs restore vascular network in oxygen-induced retinopathy. (A and B) Culture supernatant from 3D gel culture repairs the oxygen-induced retinopathy (OIR) model's phenotype, and its rescue effect is regulated by CD44. (A) Confocal image of GFP-ECFC in collagen gel culture. (B) qPCR array gene profile analysis for human angiogenic growth factors expressed on ECFCs-scrRNA or ECFCs-shCD44 cultured in 3D gel for 48 hours. $n=3$. Genes dysregulated by $>1.5$-fold with $P$ values of less than 0.05 were plotted. (C) Angiogenic protein arrays of conditioned media from 3D gel culture of control ECFCs (ECFCs-scrRNA) (A and B) or CD44 knockdown ECFCs (ECFCsshCD44). Boxes outlined with various colors indicate ECFC-secreted angiocrine factors. (D) ELISA analysis for human IL-8, IGFBP2, and IGFBP3 in conditioned media from 3D gel culture of ECFCs-scrRNA or ECFCs-shCD44. $n=7$. Student's $t$ test. (E) Representative GS lectin-stained flat-mount retinas and (F) quantification for eyes harvested at P17 after injection at P12 with cell culture supernatant from 3D gel culture of control ECFCs (ECFCs-scrRNA), CD44 knockdown ECFCs (ECFCs-shCD44), or gel without ECFCs in OIR. ${ }^{*} P<0.05$, Kruskal-Wallis test with Dunn's multiple comparison test. $n=16-18$. Scale bar: $500 \mu \mathrm{m}$. Error bars represent SD in $\mathbf{D}$ and SEM in $\mathbf{F}$. 
A

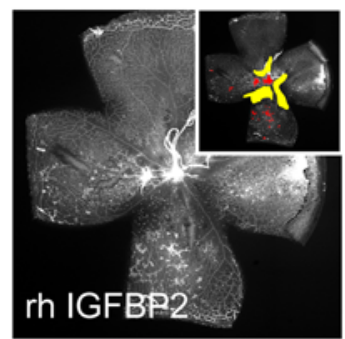

B

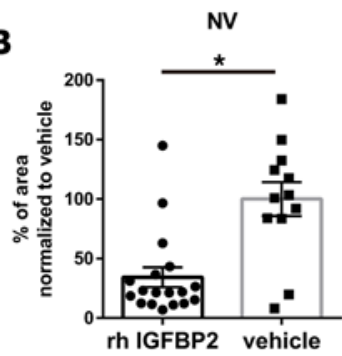

C

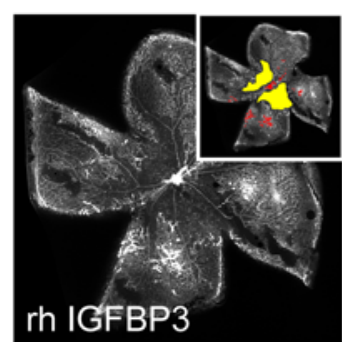

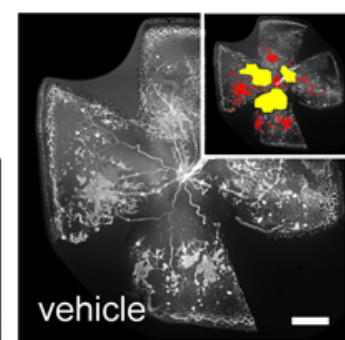
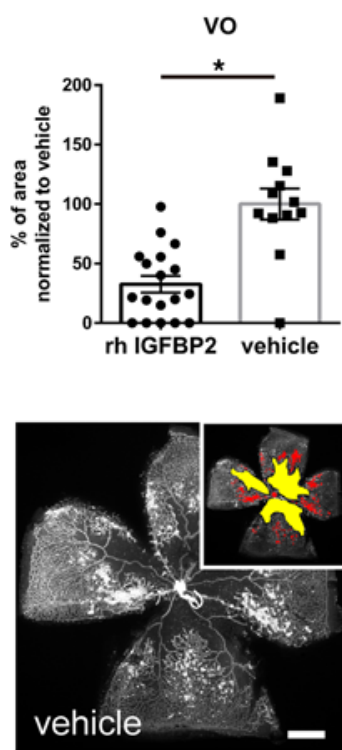

E

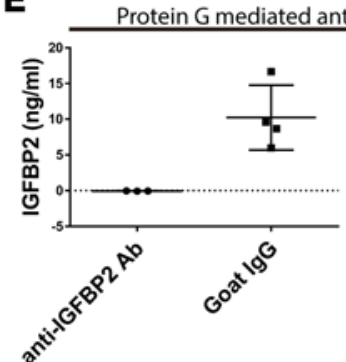

$\mathbf{F}$
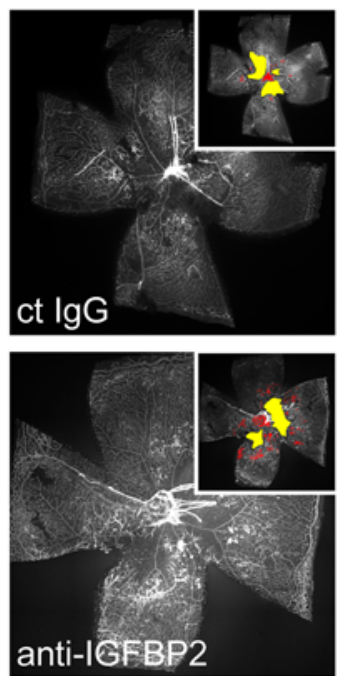
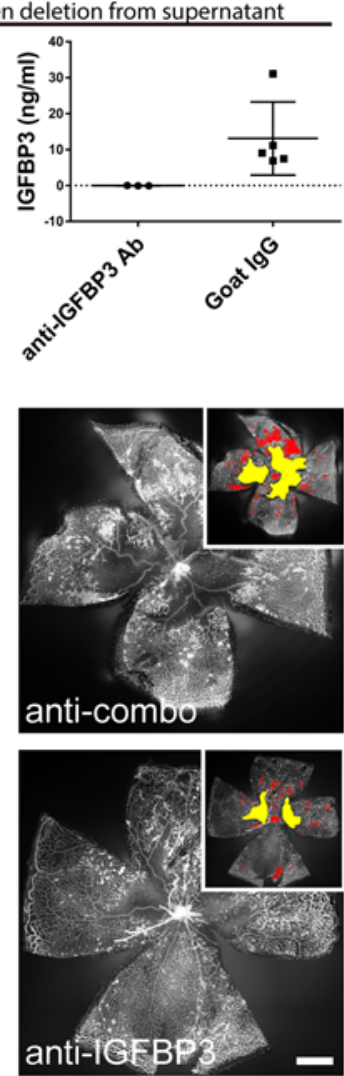

anti-1G BP3 -
D

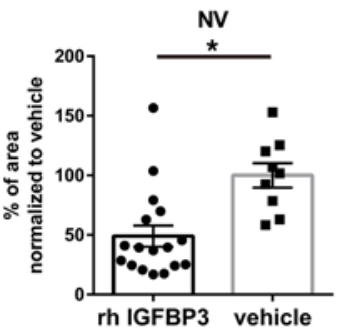

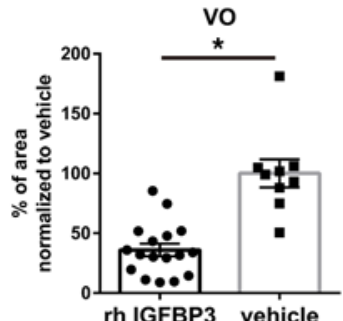

G

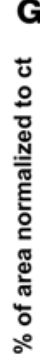

\section{NV}

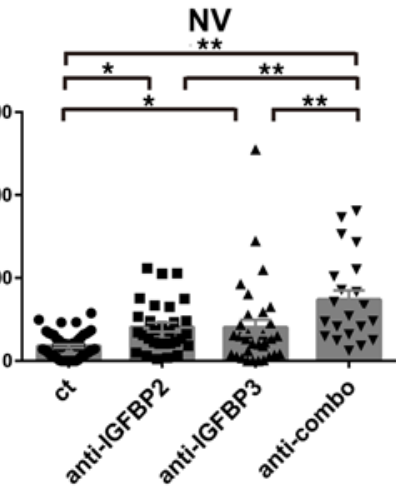

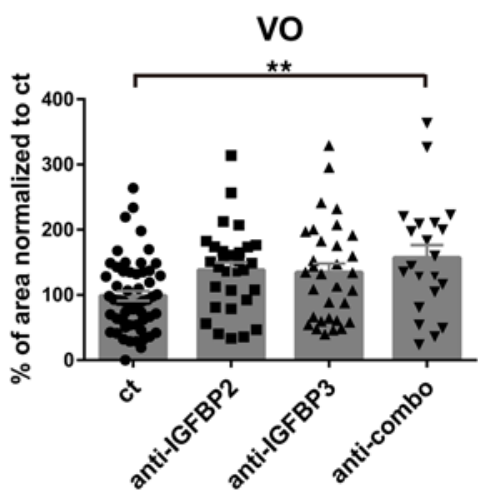

Figure 8. IGFBPs secreted from ECFCs exert reparative effect for oxygen-induced retinopathy. (A-D) Treatment with 1 ng rhIGFBP2 (A and B) or rhIGFBP3 (C and D) efficiently decreased neovascular tufts (NV) and vaso-obliterated regions (VO) at P17 when compared with vehicle-injected eyes. Results are expressed as a percentage of NV or VO area, normalized to vehicle-injected eyes. $n=9-16 .{ }^{*} P<0.05$, Mann-Whitney test. Scale bar: $500 \mu \mathrm{m}$. (E-G) Depletion of IGFBPs in cell culture supernatant from 3D gel culture of ECFCs partially cancelled the rescue effect in oxygen-induced retinopathy (OIR). (E) ELISA analysis after depletion of IGFBP2 and IGFBP3 using Protein G magnetic beads with each neutralizing antibody. (F) Representative GS lectin-stained flatmount retinas and (G) quantification for eyes harvested at P17 after injection at P12 with cell culture supernatant treated with Protein $\mathrm{G}$ magnetic beads with neutralizing antibody either of IGFBP2 or IGFBP3 and both (combo-Ab) from 3D gel culture of ECFCs in OIR. $n>21$. ${ }^{*} P<0.05$, ${ }^{* *} P<0.01$, Kruskal-Wallis test with Dunn's multiple comparison test. Error bars represent SEM. Scale bar: $500 \mu \mathrm{m}$.

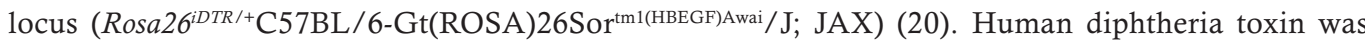
administrated as previously described (48). Pde6b $b^{\text {rd10/rd10 }}(\mathrm{rd} 10)$ mice (B6.CXB1-Pde6b $\mathrm{b}^{\text {rd10 }} / \mathrm{J}$ ) and Rag2 knockout mice (C.B6(Cg)-Rag2 $2^{\mathrm{tm} 1.1 \mathrm{cgn}} / \mathrm{J}$, no. 008448) were also purchased from JAX.

Cell preparation and culture. Human ECFCs were derived from umbilical cord blood (UCB) obtained after full-term deliveries and cultured essentially as described previously (27). Namely, UCB was collected in PBS with $20 \mu \mathrm{g} / \mathrm{ml}$ heparin (STEMCELL Technologies, 07980), and the processing of UCB samples was started 
within 6 hours after delivery in all cases. Mononuclear cells were isolated from UCB by Lymphoprep (STEMCELL Technologies, 07851), resuspended into complete EGM-2 medium (SCM) (EGM-2 bullet kit, Lonza, cc-3202, and 10\% FBS) or XFM provided by STEMCELL Technologies, and seeded onto 6-well plates coated with rat collagen type 1 (Corning, 354236). In the case of XFM cell culture, we used ACF attachment substrate, provided by STEMCELL Technologies. The medium was changed daily for 7 days and then every other day. Individual ECFC colonies emerged 4-16 days after seeding and were replated separately. We performed cloning assays in 96-well plates, and colonies were counted after fixation and staining. Individual ECFC colonies were analyzed by flow cytometry for the expression of endothelial and hematopoietic cell surface markers. To understand the precise mechanism underlying the reparative effect of ECFCs, we performed all subsequent experiments after Figure 1 with ECFCs cultured with EGM supplemented with 10\% FBS. For gel culture of ECFCs, a collagen solution (pig acid soluble form, Cellmatrix type I-A) was obtained from Nitta Gelatin and used as previously described (49). Cultured ECFCs were added to the collagen suspension to a final concentration of 2 million cells/ml collagen. $500 \mu \mathrm{l}$ of the collagen/cell suspension was added into the wells of a Costar 24-well tissue culture dish (Costar Corp.) and left at room temperature for 15 minutes to permit collagen polymerization. Following gel formation, $1 \mathrm{ml}$ of medium was added per well, which allowed the cells to be fed from the top of the collagen gel. For supernatant experiments, samples of conditioned medium were collected from gel culture of ECFCs in EBM-2 with 10\% FBS for first 24 hours and media were changed to EBM-2 for next 24 hours to collect supernatant. All experiments were performed with individual ECFC colonies between passage 4 and 7. All experiments were performed with at least 3 biologically independent ECFC colonies, originating from 3 distinct cord blood donors.

Lentiviral-mediated GFP expression and shRNA knockdown. For labeling ECFCs, we used GFP (CMV-Neo) lentiviral particles (GenTarget Inc.). Lentiviral-shRNA clones were also generated by GenTarget Inc. For lentivirus infection, cells were selected by flow cytometry. Human standard CD44 was silenced by treatment with combined shRNA clones TRCN0000308110 and TRCN0000296190

Intravitreal injection. In one eye of each animal, $5 \times 10^{4}$ cells for OIR model mice or $1 \times 10^{5}$ cells for RD10 mice in $0.5 \mu \mathrm{l}$ vehicle (Dulbecco's PBS containing $0.5 \%$ BSA and $2 \mathrm{mM}$ EDTA) were injected into the vitreous using a Hamilton syringe fitted with a 33-gauge needle (Hamilton) at P12. In some cases, we injected at P7, as indicated in Figure 2. In the contralateral eye, we injected an equal number of control cells or vehicle alone. For supernatant experiments, collected media were filtered $(0.2 \mu \mathrm{m})$ and then concentrated 100 -fold (Amicon Ultra centrifugal filters; 3-kDa cutoff, Millipore). For recombinant protein experiments, $1 \mathrm{ng}$ of human IGFBP2 or IGFBP3 (R\&D) was injected. To deplete IGFBPs in supernatant, we used an immunoprecipitation kit with Dynabeads Protein G (Thermo Fisher Scientific) following the manufacturer's instruction. Briefly, we incubated the IGFBP2 and IGFBP3 blocking antibody or goat IgG (R\&D) with Dynabeads. We removed the Dynabeads supernatant using a magnet to pull it into a pellet and then added the 3D culture supernatant, which was obtained as described above. Finally, we collected the supernatant, using the magnet to immunoprecipitate the Dynabeads and attached protein and then injected it into OIR eyes after concentration.

Immunofluorescence. Whole-mount preparations for retinas were performed as previously described (50). For retinal and post-capsular flat-mounts, we dissected only the cornea, choroid, and sclera from enucleated eyes, and then we punctured the anterior capsule of the lens and removed the nuclei and cortex of the lens with forceps. Immunostaining of cryosections was carried out as previously described (51). The primary antibodies used in this study are described in Supplemental Table 1. The retinas and sections were incubated with the corresponding Alexa Fluor-conjugated secondary antibodies (Invitrogen), and the nuclei were stained with Hoechst 33342 or DAPI (Vector Laboratories). TUNEL staining was performed using an In Situ Cell Death Detection Kit (Roche Diagnostics) according to the manufacturer's instructions. Perfusion of FITC dextran and FITC albumin was performed as previously described (22).

Confocal microscopy and quantification. All images were acquired with a confocal laser-scanning microscope (LSM 700 or 710, Zeiss) and processed with the ZEN 2010 software (Zeiss). OIR quantification of vaso-obliteration and neovascularization was carried out as described previously (18). In brief, the area of VO was measured by carefully delineating the avascular zones in the central retina of GS lectin-stained retinas and calculating the total area using Photoshop CS6 (Adobe). For quantification of retinal thickness in OIR eyes, the thicknesses of the IPL, INL, and ONL were measured on the photographs of central retina (about $500 \mu \mathrm{m}$ from the optic disc) in a masked fashion. 3D reconstructions were generated using ZEN 2010. For branch point quantification of the vascular plexus, $8 \times 200$ magnification images ( 4 center and 4 peripheral; $320 \times 320 \mu \mathrm{m}$ fields of view per retina) were chosen 
from each scanned image of the deep layer of the retina plexus of P32 RD10 mice, and the number of branch points obtained from each of the 8 fields was averaged. The quantification of retinal thickness and TUNEL-positive cells was performed as previously described (48).

Gene profiling, ELISA, and MSD analysis. Total RNA was isolated from cells and tissues using the RNeasy Micro Kit (QIAGEN) and reverse transcribed using the QuantiTect Reverse Transcription Kit (QIAGEN), following the manufacturer's instructions. qPCR assays were performed with the CFX96 Touch Real-Time PCR Detection System (Bio-Rad) using a PCR array (RT2 Profiler PCR Array for human angiogenic growth factor [PAHS-072Z], human or mouse neurotrophin and receptors [PAHS031Z or PAMM-031Z]) according to the manufacturer's instructions (QIAGEN). To analyze the expression of genes, RT2 Profiler PCR Array Data Analysis Suite version 3.5 was used. Proteome Profiler Human Antibody Array Kits (R\&D Systems) were used for this analysis following the manufacturer's instructions. Human IL-8, IGFBP-2, and IGFBP-3 Quantikine ELISA Kits (R\&D) were used for sandwich ELISA experiments. Each condition was tested in triplicates according to the manufacturer's specifications, and the output was measured using a microplate reader (Synergy 2; BioTek). Concentrations were obtained by fitting the data to a standard curve using nonlinear 3-factor regression. MSD analysis was performed as previously described (52). High-binding carbon electrodes were set in the bottom of microplates and MSD assays used electrochemiluminescent labels that are conjugated to detection antibodies (SULFO-TAG) and allow for sensitive detection. Electricity was applied to the plate electrodes by an MSD instrument (QuickPlex SQ 120), leading to light emission by SULFO-TAG labels. Light intensity was then measured to quantify analytes in the sample. We optimized human IGFBP2 and IGFBP3 MSD kits following the manufacturer's instructions. Total proteins from whole P14 OIR eyes were corrected with $300 \mu 1$ T-PER Lysis buffer after injection of ECFCs at P12.

Flow cytometry. ECFCs were incubated at $4^{\circ} \mathrm{C}$ for 30 to 60 minutes with varying concentrations of the primary or isotype control antibody, as outlined below in $1001 \mathrm{PBS}$ and 4\% FBS, washed 3 times, and analyzed by FACS (BD). For cell sorting for injected ECFCs, dissected corneas and scleras of at least 15 mouse neonates were incubated after injection for 30 minutes at $37^{\circ} \mathrm{C}$ in DMEM containing $1 \%$ collagenase (Wako) before cells were dissociated by gentle trituration. The stained cells were analyzed and sorted using a FACSAria flow cytometer (BD) with FlowJo (TreeStar) software. Dead cells were excluded from the analyses using the 2D profile of forward versus side scatter. Using these negative and positive control tubes, we set fluorescence voltages and the compensation matrix according to the instructions of the manufacturer. We applied these setting parameters to all samples analyzed. We used primary murine monoclonal antibodies against human CD44 antibody conjugated to APC (clone: IM-7 and G44-57, BD PharMingen) and to PE (clone: DB105, Miltenyi Biotec) for CD $44^{\mathrm{hi} / \mathrm{lo}}$ ECFC characterization. Subsequent immunolabeling was performed with G4457 clone. For phenotypic analysis of the cells, we used antibodies described in Supplemental Table 1.

Ganzfeld ERG. ERG was performed according to procedures previously described (53). Mice were dark adapted overnight before the experiments and anesthetized under a dim red light by intraperitoneal injection of $15 \mathrm{mg} / \mathrm{kg}$ ketamine and $7 \mathrm{mg} / \mathrm{kg}$ xylazine. Silver needle electrodes served as a reference (forehead) and ground (tail). Full-field ERGs were recorded from the corneal surface of each eye after pupil dilation (with $2.5 \%$ phenylephrine and $1 \%$ tropicamide) with active contact lens electrodes (Mayo). A computerized system with an electronically controlled Ganzfeld dome was used (Espion E2 with Colordome; Diagnosys). In the dark-adapted condition (scotopic), we recorded rod and mixed cone/rod responses to a series of white flashes of increasing intensities $\left(1 \times 10^{-5}\right.$ to $\left.50 \mathrm{~cd} \times \mathrm{s} / \mathrm{m}^{2}\right)$. In the light-adapted condition (photopic), with a $30 \mathrm{~cd} / \mathrm{m}^{2}$ background, cone responses to $1-\mathrm{Hz}\left(0.63-20 \mathrm{~cd} \times \mathrm{s} / \mathrm{m}^{2}\right)$ stimuli were recorded. All ERG responses were filtered at $0.3-500 \mathrm{~Hz}$, and signal averaging was applied.

Statistics. Statistical analyses were carried out using PRISM (version 6, GraphPad Software). Experiments involving two groups were compared using unpaired, 2-tailed $t$ tests. Multiple comparisons were made using 1-way ANOVA with Tukey analysis. The normal distribution of all data sets was tested, and, depending on the results, nonparametric analyses (Mann-Whitney test or Kruskal-Wallis test with Dunn's multiple comparison test) were performed. $P<0.05$ was considered statistically significant.

Study approval. All animal experimental procedures were approved by The Scripps Research Institute Animal Care and Use Committee. All experiments were performed in accordance with the NIH Guide for the Care and Use of Laboratory Animals (National Academies Press. 2011). Informed consent was obtained from adult donors for cord blood in accordance with the Declaration of Helsinki. The Institutional Review Board at The Scripps Research Institute and Scripps Memorial Hospital La Jolla approved all protocols. 


\section{Author contributions}

SS designed experiments, performed experiments, and wrote and edited the manuscript. VM designed complement experiments, performed experiments, reviewed and edited the manuscript, and developed reagents used in this study. EA performed experiments. KL performed experiments and developed reagents used in this study. YU, SM, and FB provided advice on retinal experiments and edited the manuscript. JKT collected blood samples. RF conducted experiments and assisted in preparation of the manuscript. RW and CP supervised the xeno-free culture work, performed experiments, reviewed and edited the manuscript, and developed reagents used in this study. ELS and PDW supervised retinal work and reviewed and edited the manuscript. MF supervised the work and reviewed and edited the manuscript.

\section{Acknowledgments}

We would like to thank Michael Dorrell, Kevin Eade, and Marin Gantner (Lowy Medical Research Institute) as well as Daniel Feitelberg, Maki Kitano, Mauricio Rosenfeld, Kristen Slanina, Matthew Haynes, Brian Seegers, and Sarah Meadows Carey (The Scripps Research Institute) for technical and administrative assistance or critical discussions of the data. This work was supported by grants to MF from the National Eye Institute (R01 EY11254 and R24 EY022025) and the Lowy Medical Research Foundation (MacTel). SS was supported by a fellowship from the Japan Society for the Promotion of Science and the Uehara Memorial Foundation.

Address correspondence to: Martin Friedlander, The Scripps Research Institute, 10550 N. Torrey Pines Road, La Jolla, California 92037, USA. Phone: 858.784.9138; E-mail: friedlan@scripps.edu.

PDW's present address is: Department of Ophthalmology, Baylor College of Medicine, Houston, Texas, USA.

1. Grunwald JE, Maguire AM, Dupont J. Retinal hemodynamics in retinitis pigmentosa. Am J Ophthalmol. 1996;122(4):502-508

2. Birch DG, Weleber RG, Duncan JL, Jaffe GJ, Tao W, Ciliary Neurotrophic Factor Retinitis Pigmentosa Study Groups. Randomized trial of ciliary neurotrophic factor delivered by encapsulated cell intraocular implants for retinitis pigmentosa. Am J Ophthalmol. 2013;156(2):283-292.e1.

3. Ingram DA, et al. Identification of a novel hierarchy of endothelial progenitor cells using human peripheral and umbilical cord blood. Blood. 2004;104(9):2752-2760.

4. Kang KT, Coggins M, Xiao C, Rosenzweig A, Bischoff J. Human vasculogenic cells form functional blood vessels and mitigate adverse remodeling after ischemia reperfusion injury in rats. Angiogenesis. 2013;16(4):773-784.

5. Moubarik C, et al. Transplanted late outgrowth endothelial progenitor cells as cell therapy product for stroke. Stem Cell Rev. 2011;7(1):208-220.

6. Medina RJ, O'Neill CL, Humphreys MW, Gardiner TA, Stitt AW. Outgrowth endothelial cells: characterization and their potential for reversing ischemic retinopathy. Invest Ophthalmol Vis Sci. 2010;51(11):5906-5913.

7. Prasain N, et al. Differentiation of human pluripotent stem cells to cells similar to cord-blood endothelial colony-forming cells Nat Biotechnol. 2014;32(11):1151-1157.

8. Schwarz TM, et al. Vascular incorporation of endothelial colony-forming cells is essential for functional recovery of murine ischemic tissue following cell therapy. Arterioscler Thromb Vasc Biol. 2012;32(2):e13-e21.

9. Palii CG, et al. Trichostatin A enhances vascular repair by injected human endothelial progenitors through increasing the expression of TAL1-dependent genes. Cell Stem Cell. 2014;14(5):644-657.

10. Lin RZ, Moreno-Luna R, Li D, Jaminet SC, Greene AK, Melero-Martin JM. Human endothelial colony-forming cells serve as trophic mediators for mesenchymal stem cell engraftment via paracrine signaling. Proc Natl Acad Sci USA. 2014;111(28):10137-10142.

11. Alphonse RS, et al. Existence, functional impairment, and lung repair potential of endothelial colony-forming cells in oxygen-induced arrested alveolar growth. Circulation. 2014;129(21):2144-2157.

12. Meyer K, Palmer JW. The polysaccharide of the vitreous humor. J Biol Chem. 1934;107(3):629-634.

13. Ponta H, Sherman L, Herrlich PA. CD44: from adhesion molecules to signalling regulators. Nat Rev Mol Cell Biol. 2003;4(1):33-45.

14. Cheng C, Yaffe MB, Sharp PA. A positive feedback loop couples Ras activation and CD44 alternative splicing. Genes Dev. 2006;20(13):1715-1720.

15. Pietras A, et al. Osteopontin-CD44 signaling in the glioma perivascular niche enhances cancer stem cell phenotypes and promotes aggressive tumor growth. Cell Stem Cell. 2014;14(3):357-369.

16. Zöller M. CD44: can a cancer-initiating cell profit from an abundantly expressed molecule? Nat Rev Cancer. 2011;11(4):254-267.

17. Stier S, et al. Osteopontin is a hematopoietic stem cell niche component that negatively regulates stem cell pool size. $J$ Exp Med. 2005;201(11):1781-1791.

18. Ritter MR, Banin E, Moreno SK, Aguilar E, Dorrell MI, Friedlander M. Myeloid progenitors differentiate into microglia and promote vascular repair in a model of ischemic retinopathy. J Clin Invest. 2006;116(12):3266-3276.

19. Reinisch A, et al. Humanized large-scale expanded endothelial colony-forming cells function in vitro and in vivo. Blood. 2009;113(26):6716-6725.

20. Smith LE, et al. Oxygen-induced retinopathy in the mouse. Invest Ophthalmol Vis Sci. 1994;35(1):101-111.

21. Westenskow PD, et al. Ras pathway inhibition prevents neovascularization by repressing endothelial cell sprouting. J Clin Invest. 2013;123(11):4900-4908. 
22. Scheppke L, et al. Retinal vascular permeability suppression by topical application of a novel VEGFR2/Src kinase inhibitor in mice and rabbits. J Clin Invest. 2008;118(6):2337-2346.

23. Streilein JW. Ocular immune privilege: therapeutic opportunities from an experiment of nature. Nat Rev Immunol. 2003;3(11):879-889

24. Bianchi ME. DAMPs, PAMPs and alarmins: all we need to know about danger. J Leukoc Biol. 2007;81(1):1-5.

25. Carmeliet P. Angiogenesis in health and disease. Nat Med. 2003;9(6):653-660.

26. Zheng Y, et al. Regulatory T-cell suppressor program co-opts transcription factor IRF4 to control T(H)2 responses. Nature. 2009;458(7236):351-356.

27. Yoder MC, et al. Redefining endothelial progenitor cells via clonal analysis and hematopoietic stem/progenitor cell principals Blood. 2007;109(5):1801-1809.

28. Blanks JC, Johnson LV. Vascular atrophy in the retinal degenerative rd mouse. J Comp Neurol. 1986;254(4):543-553.

29. Gargini C, Terzibasi E, Mazzoni F, Strettoi E. Retinal organization in the retinal degeneration 10 (rd10) mutant mouse: a morphological and ERG study. J Comp Neurol. 2007;500(2):222-238.

30. Otani A, et al. Rescue of retinal degeneration by intravitreally injected adult bone marrow-derived lineage-negative hematopoietic stem cells. J Clin Invest. 2004;114(6):765-774.

31. Hodgkinson CP, Bareja A, Gomez JA, Dzau VJ. Emerging Concepts in Paracrine Mechanisms in Regenerative Cardiovascular Medicine and Biology. Circ Res. 2016;118(1):95-107.

32. Azar WJ, et al. IGFBP-2 enhances VEGF gene promoter activity and consequent promotion of angiogenesis by neuroblastoma cells. Endocrinology. 2011;152(9):3332-3342.

33. Das SK, et al. MDA-9/syntenin and IGFBP-2 promote angiogenesis in human melanoma. Cancer Res. 2013;73(2):844-854.

34. Lofqvist C, et al. IGFBP3 suppresses retinopathy through suppression of oxygen-induced vessel loss and promotion of vascular regrowth. Proc Natl Acad Sci USA. 2007;104(25):10589-10594

35. Chang $\mathrm{KH}$, et al. IGF binding protein-3 regulates hematopoietic stem cell and endothelial precursor cell function during vascular development. Proc Natl Acad Sci USA. 2007;104(25):10595-10600.

36. Baxter RC. IGF binding proteins in cancer: mechanistic and clinical insights. Nat Rev Cancer. 2014;14(5):329-341.

37. Pereira JJ, et al. Bimolecular interaction of insulin-like growth factor (IGF) binding protein-2 with alphavbeta 3 negatively modulates IGF-I-mediated migration and tumor growth. Cancer Res. 2004;64(3):977-984.

38. Wang GK, Hu L, Fuller GN, Zhang W. An interaction between insulin-like growth factor-binding protein 2 (IGFBP2) and integrin alpha5 is essential for IGFBP2-induced cell mobility. J Biol Chem. 2006;281(20):14085-14091.

39. Kobayashi $\mathrm{H}$, et al. Angiocrine factors from Akt-activated endothelial cells balance self-renewal and differentiation of haematopoietic stem cells. Nat Cell Biol. 2010;12(11):1046-1056.

40. Rafii S, Butler JM, Ding BS. Angiocrine functions of organ-specific endothelial cells. Nature. 2016;529(7586):316-325

41. Travis GH. Mechanisms of cell death in the inherited retinal degenerations. Am J Hum Genet. 1998;62(3):503-508

42. Higashi Y, Sukhanov S, Anwar A, Shai SY, Delafontaine P. IGF-1, oxidative stress and atheroprotection. Trends Endocrinol Metab. 2010;21(4):245-254.

43. Ramsden CM, Powner MB, Carr AJ, Smart MJ, da Cruz L, Coffey PJ. Stem cells in retinal regeneration: past, present and future. Development. 2013;140(12):2576-2585.

44. Hur J, et al. Characterization of two types of endothelial progenitor cells and their different contributions to neovasculogenesis. Arterioscler Thromb Vasc Biol. 2004;24(2):288-293.

45. Karin M, Clevers H. Reparative inflammation takes charge of tissue regeneration. Nature. 2016;529(7586):307-315.

46. Fukuda S, et al. Functional endothelial progenitor cells selectively recruit neurovascular protective monocyte-derived F4/80(+) /Ly6c(+) macrophages in a mouse model of retinal degeneration. Stem Cells. 2013;31(10):2149-2161.

47. McKee CM, et al. Hyaluronan (HA) fragments induce chemokine gene expression in alveolar macrophages. The role of HA size and CD44. J Clin Invest. 1996;98(10):2403-2413.

48. Usui Y, et al. Neurovascular crosstalk between interneurons and capillaries is required for vision. J Clin Invest. 2015;125(6):2335-2346.

49. Ilan N, Mahooti S, Madri JA. Distinct signal transduction pathways are utilized during the tube formation and survival phases of in vitro angiogenesis. J Cell Sci. 1998;111(Pt 24):3621-3631.

50. Sakimoto S, et al. An angiogenic role for adrenomedullin in choroidal neovascularization. PLoS One. 2013;8(3):e58096.

51. Sakimoto S, et al. A role for endothelial cells in promoting the maturation of astrocytes through the apelin/APJ system in mice. Development. 2012;139(7):1327-1335.

52. Regeniter A, et al. Biomarkers of dementia: comparison of electrochemiluminescence results and reference ranges with conventional ELISA. Methods. 2012;56(4):494-499.

53. Kurihara T, et al. Hypoxia-induced metabolic stress in retinal pigment epithelial cells is sufficient to induce photoreceptor degeneration. Elife. 2016;5:e14319. 